\title{
Prehomogeneous spaces for Borel subgroups of general linear groups
}

\author{
Simon M. Goodwin and Lutz Hille
}

October 15,2018

\begin{abstract}
Let $k$ be an algebraically closed field. Let $B$ be the Borel subgroup of $\mathrm{GL}_{n}(k)$ consisting of nonsingular upper triangular matrices. Let $\mathfrak{b}=$ Lie $B$ be the Lie algebra of upper triangular $n \times n$ matrices and $\mathfrak{u}$ the Lie subalgebra of $\mathfrak{b}$ consisting of strictly upper triangular matrices. We classify all Lie ideals $\mathfrak{n}$ of $\mathfrak{b}$, satisfying $\mathfrak{u}^{\prime} \subseteq \mathfrak{n} \subseteq \mathfrak{u}$, such that $B$ acts (by conjugation) on $\mathfrak{n}$ with a dense orbit. Further, in case $B$ does not act with a dense orbit, we give the minimal codimension of a $B$-orbit in $\mathfrak{n}$. This can be viewed as a first step towards the difficult open problem of classifying of all ideals $\mathfrak{n} \subseteq \mathfrak{u}$ such that $B$ acts on $\mathfrak{n}$ with a dense orbit.

The proofs of our main results require a translation into the representation theory of a certain quasi-hereditary algebra $\mathcal{A}_{t, 1}$. In this setting we find the minimal dimension of $\operatorname{Ext}_{\mathcal{A}_{t, 1}}^{1}(M, M)$ for a $\Delta$-good $\mathcal{A}_{t, 1}$-module of certain fixed $\Delta$-dimension vectors.
\end{abstract}

\section{Introduction}

Let $k$ be an algebraically closed field. Let $P$ be a parabolic subgroup of $\mathrm{GL}_{n}(k)$, write $\mathfrak{p}$ for the Lie algebra of $P$ and $\mathfrak{p}_{u}$ for the Lie algebra of the unipotent radical of $P$. The group $P$ acts on $\mathfrak{p}_{u}$ via conjugation, and this induces an action of $P$ on any Lie ideal $\mathfrak{n}$ of $\mathfrak{p}$ contained in $\mathfrak{p}_{u}$. Thanks to a classical result of Richardson $(\underline{\mathrm{Rc}})$ the parabolic group $P$ always acts on $\mathfrak{p}_{u}$ with a dense orbit. However, there is not always a dense $P$-orbit in $\mathfrak{n}$, see HR3 for a counter example in case $\mathfrak{n}$ is the derived subalgebra $\mathfrak{p}_{u}^{\prime}=\left[\mathfrak{p}_{u}, \mathfrak{p}_{u}\right]$ of $\mathfrak{p}_{u}$.

Now consider the case $P=B$ is a Borel subgroup of $\mathrm{GL}_{n}(k)$. We write $U$ for the unipotent radical of $B$. The Lie algebras of $B$ and $U$ are denoted by $\mathfrak{b}$ and $\mathfrak{u}$ respectively. We may take $B$ to be the group of invertible upper triangular $n \times n$ matrices. Then $U$ consists of the upper unitriangular $n \times n$ matrices, $\mathfrak{b}$ is the Lie algebra of upper triangular $n \times n$ matrices, and $\mathfrak{u} \subseteq \mathfrak{b}$ consists of the strictly upper triangular matrices. Let $\mathfrak{n}$ be an ideal of $\mathfrak{b}$ satisfying $\mathfrak{u}^{\prime} \subseteq \mathfrak{n} \subseteq \mathfrak{u}$. The principal result of this paper gives a classification of all pairs $(B, \mathfrak{n})$ such that $\mathfrak{n}$ is a prehomogeneous space for $B$. We recall that $\mathfrak{n}$ is called a prehomogeneous space for $B$ provided $B$ acts on $\mathfrak{n}$ with a dense orbit. This result can be viewed 
as a first step towards classifying all ideals $\mathfrak{n}$ of $\mathfrak{b}$ contained in $\mathfrak{u}$ such that $B$ acts on $\mathfrak{n}$ with a dense orbit. This classification is known to be a difficult problem, but we believe that this paper contains many ideas and techniques for attacking this problem.

Let $N$ be the normal subgroup of $B$ such that $U^{\prime} \subseteq N \subseteq U$, and let $\mathfrak{n}=$ Lie $N$. We note that if $x \in \mathfrak{n}$ is a representative of a dense $B$-orbit in $\mathfrak{n}$, then $1+x$ is a representative of a dense $B$-conjugacy class in $N$. Therefore, our classification of when $B$ acts on $\mathfrak{n}$ with a dense orbit gives a classification of when there is a dense $B$-conjugacy class in $N$.

For $x \in \mathfrak{u}$, the orbit map $B \rightarrow B \cdot x$ is separable; this follows from Bo Prop. 6.7] and the fact that $C_{B}(x)=1+\mathfrak{c}_{\mathfrak{b}}(x)$. Therefore, our classification of when $\mathfrak{n}$ is a prehomogeneous space for $B$ leads to a classification of when there exists $x \in \mathfrak{n}$ such that $[\mathfrak{b}, x]=\mathfrak{n}$. Moreover, since the representative $x \in \mathfrak{n}$ of the dense orbit (when it exists) that we construct in Section [5] is a matrix whose entries are all 0 or 1 , this classification remains valid if we replace $k$ by an arbitrary field.

To formulate our main result we need to associate to the $B$-module $\mathfrak{n}$ a sequence of positive natural numbers $\underline{a}=\left(a_{0}, a_{1}, \ldots, a_{r}, a_{r+1}\right)$. The idea is that $\mathfrak{n}$ is determined by which elementary matrices $e_{i, i+1}$ it contains. Let $\left\{i_{0}, \ldots, i_{r}\right\}$ be the set of $i$ such that $e_{i, i+1} \in \mathfrak{n}$, where $i_{j}<i_{j+1}$ for each $j$. Then we define $\underline{a}$ by: $a_{0}=i_{0} ; a_{j}=i_{j}-i_{j-1}$, for $j=1, \ldots, r$; and $a_{r+1}=n-i_{r}$. We refer the reader to Section 2 for an alternative definition of $\underline{a}$ and an example.

We define $\operatorname{cd}(B ; \mathfrak{n})$ to be the codimension of a $B-$ orbit of maximal dimension in $\mathfrak{n}$. In our main theorem below, as well as giving a classification of all instances when $B$ acts on $\mathfrak{n}$ with a dense orbit, we give the value of $\operatorname{cd}(B ; \mathfrak{n})$ when $B$ does not act with a dense orbit. In fact our proof constructs a family of $B$-orbits of maximal dimension whose union is dense in $\mathfrak{n}$.

Theorem 1.1. Let $B$ be the Borel subgroup of $\mathrm{GL}_{n}(k)$ consisting of upper triangular matrices. Let $\mathfrak{n}$ be a Lie ideal of $\mathfrak{b}$ satisfying $\mathfrak{u}^{\prime} \subseteq \mathfrak{n} \subseteq \mathfrak{u}$ and associate the sequence $\underline{a}$ to $\mathfrak{n}$ as above. Then:

(1) $B$ acts on $\mathfrak{n}$ with a dense orbit precisely when the number of indices $i$ with $1 \leq i \leq r$ and $a_{i}$ even is at most one.

(2) If $B$ does not act on $\mathfrak{n}$ with a dense orbit, then the minimal codimension of a $B$-orbit in $\mathfrak{n}$ is

$$
\operatorname{cd}(B ; \mathfrak{n})=\sharp\left\{i \mid 1 \leq i \leq r, a_{i} \text { is even }\right\}-1 .
$$

The proof of the theorem uses methods both from algebraic group theory, and from representation theory of algebras. More specifically we use algebraic group theoretic techniques to show that certain ideals $\mathfrak{n}$ do not have a dense orbit, or the codimension of a $B$-orbit of maximal dimension is at least the claimed value. In particular, we use results of the first author from Gd2 that give unique minimal representatives of $B$-orbits in $\mathfrak{n}$. This is necessary in order to show that elements in the families that we construct are pairwise non-conjugate. For the converse we use representation theoretic methods as explained in the next paragraph. 
It was shown and intensively used in a series of papers that orbits for parabolic group actions are related to modules over certain quasi-hereditary algebras (see e.g. [HR2, [BH1, [BHRR], and [BHRZ]). In our case we have to deal with the algebra $\mathcal{A}_{t, 1}$. It was first considered in detail in BH1] (see Section 2 for a definition and relevant properties). For each element $x \in \mathfrak{n}$ there is a corresponding $\mathcal{A}_{t, 1}$-module $M(x)$, so that elements in the same $B$-orbit define isomorphic $\mathcal{A}_{t, 1}$-modules. For our proofs a representation-theoretic characterization of a dense orbit is crucial: an element $x$ in $\mathfrak{n}$ represents a dense orbit precisely when $\operatorname{Ext}^{1}(M(x), M(x))=0$ (Theorem 3.1). This allows us to construct a dense orbit explicitly using certain well-known standard $\mathcal{A}_{t, 1}$-modules, see Sections 4 Further, we show in Theorem 3.1 that the codimension of a $B$-orbit in $\mathfrak{n}$ is equal to the dimension of $\operatorname{Ext}^{1}(M(x), M(x))$. This result is of importance for our construction of a family of $B$-orbits whose union is dense in $\mathfrak{n}$.

As hinted at above there has been a lot of recent interest in the action of a parabolic subgroup $P$ of a reductive algebraic group $G$ on the Lie algebra $\mathfrak{p}_{u}$ of its unipotent radical. For example, there is a classification of all instances when $P$ acts on $\mathfrak{p}_{u}$ with finitely many orbits, see [HR2] and [JR]. Further, for $G$ not of type $E_{7}$ or $E_{8}$, there is a classification of all instances when $P$ acts on higher terms $\mathfrak{p}_{u}^{(l)}$ of the descending central series of $\mathfrak{p}_{u}$ with a finite number of orbits, see [BH1], BHRö] and [GR2].

There has also been much interest in the question of when $\mathfrak{p}_{u}^{(l)}$ is a prehomogeneous space for $P$. This was first investigated by G. Röhrle and the second author in HR3. It was considered further by G. Röhrle and the first author in GR1 and GR2. In particular, the results of these papers give a classification of all instances when a Borel subgroup $B$ acts of $\mathfrak{u}^{(l)}$ with a dense orbit. Further, in Gd1 the first author describes a computer program that determines when a Borel subgroup $B$ acts on any $B$-submodule of $\mathfrak{u}$ with a dense orbit.

There has been other interest in the adjoint orbits of a Borel subgroup $B$ of a reductive algebraic group on $\mathfrak{u}$. The problem of determining the $B$-orbits (and $U$-orbits) in $\mathfrak{u}$ was addressed by H. Bürgstein and W.H. Hesselink in BüHe]. They were motivated by the problem of describing the component configuration of the Springer fibre $\mathcal{B}_{x}=\{B \in \mathcal{B}: x \in \operatorname{Lie} B\}$ where $\mathcal{B}$ denotes the variety of Borel subgroups of $G$ and $x \in \mathfrak{g}$ is nilpotent, see $\$ 1.4$ of loc. cit. The first author studied the $B$-orbits and $U$-orbits in $\mathfrak{u}$ in Gd2. In particular, in loc. cit. it is shown that any $U$-orbit contains a unique so-called minimal representative.

In case $G$ is a reductive algebraic group defined and split over the finite field $\mathbb{F}_{q}$ of $q$ elements ( $q=p^{s}$, where $p$ is prime), there has been much interest in the conjugacy classes of a Sylow $p$-subgroup of the Chevalley group $G(q)$. If $B$ is a Borel subgroup of $G$ that is defined over $\mathbb{F}_{q}$, then $U(q)$ is a Sylow $p$-subgroup of $G$. For the case $G=\mathrm{GL}_{n}(k)$, we refer the reader to $\mathrm{Hg}$, [Rb], $\mathrm{Th}$ ] and $\mathrm{VA}$; for arbitrary $G$, see Gd2.

In a final section of this paper we give an interpretation of Theorem 1.1 in the corresponding finite group setting. In Theorem 8.1 we give the maximal size of a $B(q)$-conjugacy class in a normal subgroup $N(q)$ of $B(q)$ such that 
$U^{\prime}(q) \subseteq N(q) \subseteq U(q)$. The proof of this theorem requires results from Gd2.

We now outline the structure of this paper. After giving some notation below we cover the requisite preliminaries in Section 2 We give the basic notation we use for the action of $B$ on $\mathfrak{n}$ and then we discuss the algebras $\mathcal{A}_{t, l}$. In particular, we give some results for calculating Hom-groups and $\mathrm{Ext}^{1}$-groups for standard $\mathcal{A}_{t, 1}$-modules that we require in the sequel. We explain the relationship between $P$-orbits in $\mathfrak{p}_{u}$ with $\Delta$-good modules for the algebra in $\mathcal{A}_{t, l}$ and prove Theorem 3.1 in Section 3. Next in Section 4 we prove one direction of Theorem 1.1(1), by constructing certain $\Delta$-good $\mathcal{A}_{t, 1}$-modules without self-extensions. We give a construction of a representative of the dense $B$-orbit in $\mathfrak{n}$ in Section $[5$ and prove that it is minimal in the sense of Gd2; the required terminology and results from loc. cit. are recalled. These representatives are required for our proof, in Section [6 that there is not a dense $B$-orbit in $\mathfrak{n}$ in the cases stated in Theorem 1.11(1). In Section 7 we prove Theorem 1.11(2). This is achieved by first constructing certain $\Delta$-good $\mathcal{A}_{t, 1}$-modules $M$ for which we can give an upper bound on the dimension of $\operatorname{Ext}^{1}(M, M)$. Then we construct a family $\mathcal{F}$ of elements of $\mathfrak{n}$ that are pairwise non-conjugate and for which we can bound the dimension of $\operatorname{Ext}^{1}(M(x), M(x))$, where $x$ is a geometric fibre of $\mathcal{F}$. Dimension arguments allow us to show that the family of $B$-orbits given by $\mathcal{F}$ has dense union in $\mathfrak{n}$ completing the proof of Theorem[1.12). The final section is dedicated to proving Theorem 8.1

\section{Basic Notation and references}

With $\subset$ we denote the strict inclusion and with $\sharp$ the number of elements in a finite set. Given $x \in \mathbb{R}$ we write $\lfloor x\rfloor$ for the greatest integer less than $x$.

We always work over an algebraically closed field $k$. We write $k^{\times}$for the nonzero elements of $k$. All algebras are $k$-algebras with unit and modules are finitely generated. We denote by $\otimes$ the tensor product over $k$. If we consider families of modules or families of elements we work over finitely generated commutative $k$-algebras. We call such an algebra an affine $k$-algebra; it is, in general, not reduced.

An affine family $\mathcal{M}$ of $\mathcal{A}$-modules is just an $\mathcal{A}-R$-bimodule, locally free over $R$, where in our situation $R$ is an affine $k$-algebra and $\mathcal{A}$ is a finite dimensional $k$-algebra. Given a maximal ideal $m$ of $R$, we have an $\mathcal{A}$-module $\mathcal{M}(m)=$ $\mathcal{M} \otimes_{R} R / m$. In this way $\mathcal{M}$ becomes a family of modules over the maximal spectrum $\operatorname{Spec}_{\text {max }} R$ of $R$.

An affine family $\mathcal{F}$ of elements in $\mathfrak{p}_{u}$ is just an element in $\mathfrak{p}_{u} \otimes R$. If $m \subset R$ is a maximal ideal, then the projection $R \rightarrow R / m \cong k$ induces a morphism $\mathfrak{p}_{u} \otimes R \rightarrow \mathfrak{p}_{u} \otimes R / m=\mathfrak{p}_{u}$. Given $\mathcal{F} \in \mathfrak{p}_{u} \otimes R$ we write $\mathcal{F}(m)=\mathcal{F} \otimes_{R} R / m$ for its image in $\mathfrak{p}_{u}$. In this way $\mathcal{F}$ becomes a family of elements over $\operatorname{Spec}_{\max } R$.

If we write Hom or $\operatorname{Ext}^{1}$ we always consider morphisms or extensions of modules for the algebra $\mathcal{A}_{t, l}$. We write hom to denote the dimension of a Homgroup and ext $^{1}$ to denote the dimension of an Ext ${ }^{1}$-group.

For some background on path algebras and quivers we refer to [GBR and 
$\underline{\mathrm{Rn}}$. Basic results on quasi-hereditary algebras can be found in DR2. The quasi-hereditary algebras $\mathcal{A}_{t, l}$ together with certain particular modules were considered in BH1. First results concerning actions with dense orbits and tilting modules in our setting can be found in BHRR] and [HR3. As a reference for the theory of algebraic groups we refer the reader to [Bo].

\section{Preliminary results}

Let $\{0\}=V_{0} \subseteq V_{1} \subseteq V_{2} \subseteq \ldots V_{t-1} \subseteq V_{t}=V$ be a flag in the finite dimensional vector space $V$ over $k$. We define the dimension vector of this flag to be $d=$ $\left(\operatorname{dim} V_{1}, \operatorname{dim} V_{2}-\operatorname{dim} V_{1}, \ldots, \operatorname{dim} V_{t}-\operatorname{dim} V_{t-1}\right)$. We also define the dimension vector $\Sigma d=\left(\operatorname{dim} V_{1}, \ldots, \operatorname{dim} V_{t}\right)$. So we have that $(\Sigma d)_{i}=\sum_{j=1}^{i} d_{j}$. In this paper we allow also some $d_{i}$ equal zero, so the flag is not proper in general.

Let $P=P(d) \subseteq \mathrm{GL}(V)$ be the stabilizer of the flag and $\mathfrak{p}=\mathfrak{p}(d)$ the Lie algebra of $P$. The Lie algebra of the unipotent radical of $P$ is given by $\mathfrak{p}_{u}=\mathfrak{p}_{u}(d)=\left\{f \in \operatorname{End}(V) \mid f\left(V_{i}\right) \subseteq V_{i-1}\right\}$. We define the ideal $\mathfrak{p}_{u}^{(1)}=\mathfrak{p}_{u}^{(1)}(d)$ of $\mathfrak{p}$ by $\mathfrak{p}_{u}^{(1)}=\left\{f \in \operatorname{End}(V) \mid f\left(V_{i}\right) \subseteq V_{i-2}\right\}$. We note that the group $P$ and the Lie algebra $\mathfrak{p}_{u}$ do not depend on how many zeros we include in $d$. However, the ideal $\mathfrak{p}_{u}^{(1)}$ differs if we insert zeros in $d$.

In this note we are interested in dimension vectors $d$ with $d_{i}=0$ or $d_{i}=1$, for all $i$; such a dimension vector is called thin. For thin dimension vectors we have that $P=B$ is a Borel subgroup of $\operatorname{GL}(V)$ and we write $\mathfrak{u}=\mathfrak{p}_{u}$ and $\mathfrak{n}=\mathfrak{p}_{u}^{(1)}$. It is straightforward to show that any ideal $\mathfrak{n}$ of $\mathfrak{b}$ satisfying $\mathfrak{u}^{\prime} \subseteq \mathfrak{n} \subseteq \mathfrak{u}$, can be obtained in this manner for some $d$, see [H], Satz 1.4.2].

In case $d$ is a thin dimension vector, we can make the following assumptions on $d$ without affecting $B$ and $\mathfrak{n}: d_{1}=1 ; d_{t}=1$; and if $d_{i}=0$, then $d_{i+1}=1$. So we make these assumptions for the rest of the paper without loss of generality. In the definition below we associate to a thin dimension vector, the sequence $\underline{a}$ counting the lengths of the strings of 1 in $d$; we note that this definition of $\underline{a}$ is equivalent to that given in the introduction.

Definition 2.1. Let $d$ be a thin dimension vector. We define the sequence $\underline{a}=\underline{a}(d)=\left(a_{0}, a_{1}, \ldots, a_{r}, a_{r+1}\right)$ of positive integers by setting $a_{i}$ to be the length of the $i$ th string of ones in $d$. That is $a_{0}+1$ is the position of the first entry 0 in $d, a_{0}+a_{1}+2$ is the position of the second entry 0 in $d$, and so on. The nonnegative integer $e(d)$ is defined by

$$
e(d)=\sharp\left\{i \mid 1 \leq i \leq r, a_{i} \text { is even }\right\} .
$$

We note that conversely each sequence $\underline{a}=\left(a_{0}, a_{1}, \ldots, a_{r}, a_{r+1}\right)$ of positive integers defines a thin dimension vector $d$ starting with a sequence of $a_{0}$ ones followed by a zero, a sequence of $a_{1}$ ones followed by zero until the last sequence of ones of length $a_{r+1}$.

Example 2.2. We consider the case where $t=17, \operatorname{dim} V=13$ and $d=$ $(1,1,0,1,1,1,0,1,1,0,1,1,1,1,1,0,1)$. Then $r=3$ and $\underline{a}=(2,3,2,5,1)$. By Theorem 1.1 the group $B \subset \mathrm{GL}(V)$ acts on $\mathfrak{n}$ with a dense orbit. 


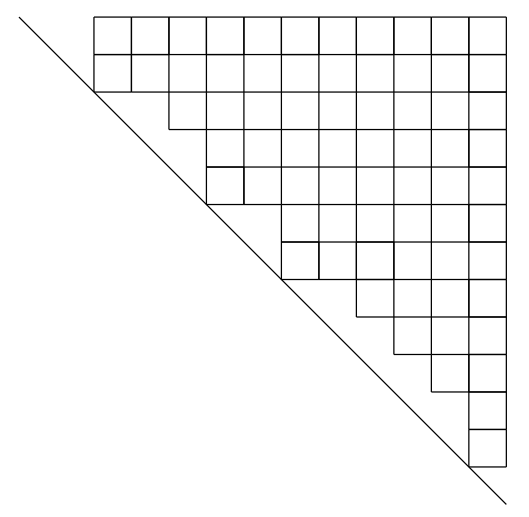

Figure 1: The ideal $\mathfrak{n}$ for $\underline{a}=(2,3,2,5,1)$

Notation 2.3. We give some notational conventions that we use throughout the sequel.

Let $d=\left(d_{1}, \ldots, d_{t}\right)$ be a thin dimension vector and let $n=\sum_{i=1}^{t} d_{i}$; as mentioned above we always assume that there are no consecutive 0 entries in $d$ and that $d_{1}=d_{t}=1$. The support of $d$ is defined by $\operatorname{supp} d=\left\{i \mid d_{i}=1\right\}$.

We define the standard flag $F(d): V_{0} \subseteq \cdots \subseteq V_{t}$ corresponding to $d$ by setting $V_{i}=k^{(\Sigma d)_{i}}$. In this way $d$ defines a Borel subgroup $B=P(d)=\operatorname{Stab}(F(d))$ of $\mathrm{GL}_{n}(k)$. Further, $d$ determines the Lie ideal $\mathfrak{n}=\mathfrak{p}_{u}^{(1)}$ of $\mathfrak{b}$. In the sequel we often refer to $B$ and $\mathfrak{n}$ as the Borel subgroup and Lie ideal corresponding to $d$.

In order to make some constructions and proofs in the sequel easier to understand we relabel the standard basis $\left\{e_{1}, \ldots, e_{n}\right\}$ of $k^{n}$ as follows: we define the function $\gamma: \operatorname{supp} d \rightarrow\{1, \ldots, n\}$ by requiring $j$ is the $\gamma(j)$ th lowest element in $\operatorname{supp} d$, and setting $f_{j}=e_{\gamma(j)}$. For $i, j \in \operatorname{supp} d$ we let $f_{i j}$ be the elementary matrix that sends $f_{i}$ to $f_{j}$ and all other $f_{i^{\prime}}$ to zero.

In the remainder of this section we discuss an algebra $\mathcal{A}_{t, l}$. In the next section we explain its connection with orbits of parabolic subgroups that was established in $\mathrm{BH} 2$. The algebras $\mathcal{A}_{t, l}$ are shown to be quasi-hereditary in loc. cit. as is reflected in the some of the terminology given below.

For positive integers $t$ and $l$ with $l<t$, we define a quiver $Q_{t, l}$ as follows: the vertex set is $\{1,2, \ldots, t\}$; and there are arrows $\alpha_{i}: i \longrightarrow i+1$ for $i=1, \ldots, t-1$ and $\beta_{j}: j+l+1 \longrightarrow j$ for $j=1, \ldots, t-l-1$. The algebra $\mathcal{A}_{t, l}$ is defined to be the quotient of the path algebra $k Q_{t, l}$ by the relations:

$$
\beta_{1} \alpha_{l+1}=0 \text { and } \beta_{i+1} \alpha_{i+l+1}=\alpha_{i} \beta_{i} \text {, for } i=1, \ldots, t-l-2 .
$$

We identify $\mathcal{A}_{t, l}$-modules with the corresponding representations of the quiver $Q_{t, l}$. So we write $M_{i}$ for the vector subspace of $M$ corresponding to the vertex $i$, and by an abuse of notation given an arrow $\gamma: i \rightarrow j$ in $Q_{t, l}$ we also write $\gamma: M_{i} \rightarrow M_{j}$ for the corresponding linear map. 


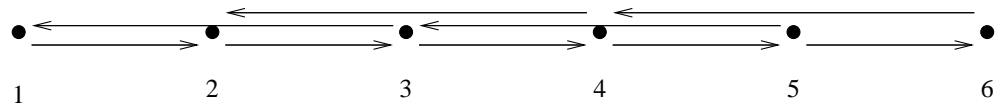

Figure 2: The quiver $Q_{6,1}$

The algebra $\mathcal{A}_{t, l}$ was introduced in $[\mathrm{BH} 2$, where it was observed to be quasihereditary. It can also be realized as a subalgebra of the endomorphism algebra of $\bigoplus_{i=0}^{t} k[T] / T^{i}$ as a $k[T]$-module. For the remainder of this section we specialize to the case $l=1$, which is the case in which we are interested in this paper; much of the discussion below generalizes easily to the case $l \geq 2$.

We need several particular $\mathcal{A}_{t, 1}$-modules. Denote by $\Delta(i)$ the representation with $\Delta(i)_{j}=k$ for $j \geq i$ and $\Delta(i)_{j}=0$ otherwise, where all the arrows $\beta$ act with zero and the arrows $\alpha$ with identity, whenever possible. The projective cover of $\Delta(i)$ is denoted by $P(i)$. Let $e_{1}, \ldots, e_{m}$ be the standard basis for $k^{m}$, for $m \in \mathbb{Z}_{\geq 0}$. Then the projective module $P(i)$ is given by: $P(i)_{j}=k^{l}$, where $l=\lfloor(j+2-i) / 2\rfloor$ if $j \geq i$ and $P(i)_{j}=0$ for $j<i$; $\alpha_{i}$ is the natural inclusion; and $\beta_{j}\left(e_{h}\right)=e_{h-1}$ if $h \geq 2$ and $\beta_{j}\left(e_{1}\right)=0$.

Let $M$ be an $\mathcal{A}_{t, 1}$-module. We denote the dimension vector of $M$ by $\operatorname{dim} M=\left(\operatorname{dim} M_{1}, \ldots, \operatorname{dim} M_{t}\right)$. A module $M$ admitting a filtration with each factor of the form $\Delta(i)$ for some $i$ is called $\Delta$-good. For such a module $M$ we define its $\Delta$-dimension vector $\operatorname{dim}_{\Delta} M=\left(\operatorname{dim} M_{1}, \operatorname{dim} M_{2}-\operatorname{dim} M_{1}, \ldots, \operatorname{dim} M_{t}-\right.$ $\left.\operatorname{dim} M_{t-1}\right)$. This vector counts the number of filtration factors isomorphic to $\Delta(i)$. For example $\operatorname{dim}_{\Delta} \Delta(i)=(0, \ldots, 0,1,0, \ldots, 0)$ and $\operatorname{dim}_{\Delta} P(i)=$ $(\ldots, 1,0,1,0,1,0,0,0, \ldots, 0,0)$. We also define the $\Delta$-support of a $\Delta$-good module $M$ as the set of all $i$ with $\left(\operatorname{dim}_{\Delta} M\right)_{i} \neq 0$.

Let $J$ be a subset of $\{1,2, \ldots, t\}$ such that for each $j \in J$ we have that $j+1 \notin J$; we call such $J$ a standard subset of $\{1, \ldots, t\}$. Let $J=\left\{j_{1}, \ldots, j_{r}\right\}$ be standard subset of $\{1, \ldots, t\}$, where $j_{i}<j_{i+1}$ for each $i$. Then there exists a unique indecomposable $\Delta$-good module $\Delta(J)$ with a thin $\Delta$-dimension vector and $\Delta$-support $J$ called a standard module; $\Delta(J)$ is defined by: $\Delta(J)_{i}=k^{l}$, where $l$ satisfies $j_{l} \leq i<j_{l+1}$ (by convention we set $j_{0}=0$ and $j_{r+1}=t+1$ ); $\alpha_{i}$ is the natural inclusion; and $\beta_{j}\left(e_{h}\right)=e_{h-1}$ if $h \geq 2$ and $\beta_{j}\left(e_{1}\right)=0$. So if $J$ is of the form $J=\{\ldots, i-4, i-2, i\}$, then we have $\Delta(J)=P(i)$, and if $J=\{i\}$, then $\Delta(J)=\Delta(i)$.

In the following theorem we collect some properties of $\Delta$-good modules for $\mathcal{A}_{t, 1}$. Parts (1) and (2) of Theorem 2.4 can be found in BH1. The last claim (3) is a generalization of BHRR Prop. 1]; it is also a consequence of Lemma 2.8 below.

Theorem 2.4. (1) For an $\mathcal{A}_{t, l}$-module $M$ the following conditions are equivalent

(a) $M$ is $\Delta$-good;

(b) the linear maps $\alpha_{i}: M_{i} \rightarrow M_{i+1}$ are injective for all $i=1, \ldots, t-l-1$; 
(c) the projective dimension of $M$ is at most one; and

(d) the socle of $M$ is isomorphic to a direct sum of copies of $\Delta(t)$.

(2) The projective modules $P(t-l), P(t-l-1), \ldots, P(t)$ are also injective.

(3) Let $l=1$ and let $J$ be a standard subset of $\{1, \ldots, t\}$. Then we have $\operatorname{Ext}^{1}(\Delta(J), \Delta(J))=0$.

In the sequel we need to know the dimensions of Hom-groups between standard modules. These dimensions are given in the following lemma.

Lemma 2.5. Let $J=\left\{j_{1}, \ldots, j_{r}\right\}$ and $K=\left\{k_{1}, \ldots, k_{s}\right\}$ be standard subsets of $\{1, \ldots, t\}$, where $j_{i}<j_{i+1}, k_{i}<k_{i+1}$ for each $i$. Then $\operatorname{hom}(\Delta(J), \Delta(K))$ is the greatest $m \leq s$ such that $r \geq m$ and $j_{r-i} \geq k_{m-i}$ for $i=0,1, \ldots, m-1$.

Proof. We have $\Delta(J)_{t}=k^{r}$ and $\Delta(K)_{t}=k^{s}$. We see that any homomorphism $\phi: \Delta(J) \rightarrow \Delta(K)$ is determined by the map $\phi_{t}: \Delta(J)_{t} \rightarrow \Delta(K)_{t}$ and in turn this map is determined by $\phi_{t}\left(e_{r}\right)=\sum_{i=1}^{m} b_{i} e_{i} \in \Delta(K)_{t}$, where $m$ is such that $b_{m} \neq 0$. For $\phi$ to be a homomorphism of $\mathcal{A}_{t, 1}$-modules, $\phi_{t}$ must restrict to the maps $\phi_{i}: \Delta(J)_{i} \rightarrow \Delta(K)_{i}$ for each $i$. It is easy to check that this holds if and only if $m$ must satisfy the conditions of the lemma. It follows that $\operatorname{hom}(\Delta(J), \Delta(K))$ is the greatest such $m$.

The proof of the lemma above allows us to define certain morphisms between standard modules.

Definition 2.6. Let $J=\left\{j_{1}, \ldots, j_{r}\right\}$ and $K=\left\{k_{1}, \ldots, k_{s}\right\}$ be standard subsets of $\{1, \ldots, t\}$, where $j_{i}<j_{i+1}, k_{i}<k_{i+1}$ for each $i$. Let $m \leq s$ be such that $r \geq m$ and $j_{r-i} \geq k_{m-i}$ for $i=0,1, \ldots, m-1$. We define $\phi^{k_{m}}: \Delta(J) \rightarrow \Delta(K)$ to be the unique morphism satisfying $\left(\phi^{k_{m}}\right)_{t}\left(e_{r}\right)=e_{m}$.

We give an example of the notation below.

Example 2.7. Let $t=7$, and let $J$ and $K$ be the standard subsets $\{1,3,7\}$ and $\{2,4,6\}$ respectively. A morphism $\phi: \Delta(J) \rightarrow \Delta(K)$ is determined by $\phi_{7}\left(e_{3}\right)$. We cannot have $\phi_{7}\left(e_{3}\right)=e_{3}$, because then we have $\phi_{3}\left(e_{2}\right)=e_{2}$, which is not possible, because $e_{2} \notin \Delta(K)_{3}$. The morphism $\phi^{4}$ is given by $\phi_{7}^{4}\left(e_{3}\right)=e_{2}$, $\phi_{7}^{4}\left(e_{2}\right)=e_{1}$ and $\phi_{7}^{4}\left(e_{1}\right)=0$; and the morphism $\phi^{2}$ is given by $\phi_{7}^{2}\left(e_{3}\right)=e_{1}$ and $\phi_{7}^{2}\left(e_{2}\right)=\phi_{7}^{2}\left(e_{1}\right)=0$.

We also require the following lemma regarding the vanishing of certain Ext ${ }^{1}$ groups between standard modules.

Lemma 2.8. Let $J$ and $K$ be standard subsets of $\{1, \ldots, t\}$. Assume that if $i \in K$, then $i-1 \in J$ or $i \in J$. Then $\operatorname{Ext}^{1}(\Delta(K), \Delta(J))=0$.

Proof. Since $\Delta(K)$ is filtered by the standard modules $\Delta(i)$ for $i \in K$, it is sufficient to prove the lemma for $K=\{i\}$. In this case we have a projective resolution

$$
0 \longrightarrow P(i-2) \stackrel{\psi}{\longrightarrow} P(i) \longrightarrow 0
$$


of $\Delta(i)$, where $\psi=\phi^{i-2}$. This gives rise to the long exact sequence

$$
\begin{aligned}
0 & \longrightarrow \operatorname{Hom}(\Delta(i), \Delta(J)) \longrightarrow \operatorname{Hom}(P(i), \Delta(J)) \longrightarrow \\
& \longrightarrow \operatorname{Hom}(P(i-2), \Delta(J)) \longrightarrow \operatorname{Ext}^{1}(\Delta(i), \Delta(J)) \longrightarrow 0 .
\end{aligned}
$$

Now using Lemma 2.5 and recalling that projective modules are certain standard modules, we have

$\operatorname{hom}(\Delta(i), \Delta(J))=1, \operatorname{hom}(P(i), \Delta(J))=l$ and $\operatorname{hom}(P(i-2), \Delta(J))=l-1$,

where $l=\sharp(J \cap\{1, \ldots, i\})$. We deduce that $\operatorname{Ext}^{1}(\Delta(i), \Delta(J))=0$ as required.

\section{The codimension of an $P$-orbit and $\mathrm{Ext}^{1}$}

Let $d=\left(d_{1}, \ldots, d_{t}\right)$ be a dimension vector with $\sum_{i=1}^{t} d_{i}=n$; we do not assume that $d$ is thin. Then we define the standard flag $F(d): V_{0} \subseteq \cdots \subseteq V_{t}$ in $k^{n}$, by $V_{i}=k^{(\Sigma d)_{i}}$. The parabolic subgroup $P=P(d)$ of $\mathrm{GL}_{n}(k)$ is defined as in the previous section. For each $l \geq 0$ we define the ideal $\mathfrak{p}_{u}^{(l)}(d)=\{f \in \operatorname{End}(V) \mid$ $\left.f\left(V_{i}\right) \subseteq V_{i-l-1}\right\}$.

In what follows we need to compare $P$-orbits and $\Delta$-good modules. The main theorem in this section (Theorem 3.1) compares the codimension of a $P(d)$-orbit in $\mathfrak{p}_{u}^{(l)}$ with the group of self-extensions of a corresponding $\Delta$-good $\mathcal{A}_{t, l}$-module $M(x)$.

We define $\mathcal{F}_{t, l}(\Delta)$ to be the category of $\Delta$-good finite dimensional modules for $\mathcal{A}_{t, l}$ (the parameters $t$ and $l$ are always fixed). Further we define $\mathcal{R}\left(\mathcal{F}_{t, l}(\Delta), d\right)$ to be the space of all $\Delta$-good representations of $\mathcal{A}_{t, l}$ with $\Delta$ dimension vector $d$

$$
\begin{aligned}
\mathcal{R}\left(\mathcal{F}_{t, l}(\Delta) ; d\right)=\left\{\left(\phi_{i}, \psi_{j}\right)\right. & \in \bigoplus_{i=1}^{t-1} \operatorname{Hom}\left(V_{i}, V_{i+1}\right) \oplus \bigoplus_{j=1}^{t-1-l} \operatorname{Hom}\left(V_{j+l+1}, V_{j}\right) \mid \\
& \left.\phi_{i} \text { is injective } \psi_{1} \phi_{l+1}=0, \psi_{i+1} \phi_{i+l+1}=\phi_{i} \psi_{i}\right\} .
\end{aligned}
$$

It is a scheme over $k$ and the group $\operatorname{GL}(\Sigma d)=\prod_{i=1}^{t} \operatorname{GL}\left((\Sigma d)_{i}\right)$ acts via conjugation. Note that the dimension vector of a $\Delta$-good module with $\Delta$-dimension vector $d$ is just $\Sigma d$. The orbits of the underlying affine variety under this action are in natural bijection with the isomorphism classes of $\Delta$-good $\mathcal{A}_{t, l}$-modules with $\Delta$-dimension vector $d$.

Below we outline the correspondence of $P(d)$-orbits in $\mathfrak{p}_{u}^{(l)}$ and isoclasses of $\Delta$-good modules with $\Delta$-dimension vector $d$ that was established in $\mathrm{BH} 2$.

Let $x$ be an element in $\mathfrak{p}_{u}^{(l)}(d)$ for some dimension vector $d$. We can associate to $x$ a $\Delta$-good module $M(x)$ over $\mathcal{A}_{t, l}$ of dimension vector $\Sigma d$ - equivalently an element of $\mathcal{R}\left(\mathcal{F}_{t, l}(\Delta) ; d\right)$. Note that $x$ maps $V_{i}$ to $V_{i-1-l}$. So we define $\phi_{i}$ to be the natural inclusion $V_{i} \longrightarrow V_{i+1}$ and $\psi_{i}$ the restriction of $x$ to $V_{i+l+1}$. It is obvious that the maps $\phi_{i}$ and $\psi_{j}$ satisfy the necessary conditions. 
Conversely, given $\left(\phi_{i}, \psi_{i}\right) \in \mathcal{R}\left(\mathcal{F}_{t, l}(\Delta) ; d\right)$. The map $\psi_{t-l-1}: V_{t} \longrightarrow V_{t-l-1}$ is an element in $\tilde{\mathfrak{p}}_{u}^{(l)}(d)$ for the stabilizer $\tilde{P}$ of the flag in $V=V_{t}$ defined by the images of the injections $\phi_{i}$. So the various $\Delta$-good modules $M$ define elements $x \in \tilde{\mathfrak{p}}_{u}^{(l)}(d)$ for possibly different groups $\tilde{P}$.

For an affine $k$-algebra $R$, we consider the set of $R$-valued points of the scheme $\mathcal{R}\left(\mathcal{F}_{t, l}(\Delta) ; d\right)$, which is denoted by $\mathcal{R}\left(\mathcal{F}_{t, l}(\Delta) ; d\right)(R)$. It consists of all pairs

$$
\left(\phi_{i}, \psi_{i}\right) \in\left(\bigoplus_{i=1}^{t-1} \operatorname{Hom}\left(V_{i}, V_{i+1}\right) \otimes R\right) \oplus\left(\bigoplus_{j=1}^{t-1-l} \operatorname{Hom}\left(V_{j+l+1}, V_{j}\right) \otimes R\right)
$$

satisfying the following two conditions:

(1) the maps $\phi_{i}$ are split injective; and

(2) $\psi_{1} \phi_{l+1}=0$ and $\psi_{i+1} \phi_{i+l+1}=\phi_{i} \psi_{i}$.

A family (over $R$ ) of $\Delta$-good modules is an $\mathcal{A}_{t, l}-R$-bimodule $\mathcal{M}$, so that the restriction of $\mathcal{M}$ to the directed quiver of type $\mathbb{A}_{t}$ (defined by the arrows $\alpha_{i}: i \longrightarrow i+1$, see Figure 2) consists of locally direct maps of locally free $R$-modules. This condition can also be read as follows: for each maximal ideal $m$ in $R$ there exists a localization $R_{f}$, with $f$ not in $m$, and an element $g \in$ $\mathrm{GL}(\Sigma d)\left(R_{f}\right)=\prod_{i=1}^{t} \mathrm{GL}\left((\Sigma d)_{i}\right)\left(R_{f}\right)$ so that $g \cdot\left(\mathcal{M}\left(\alpha_{i}\right)\right)$ is the standard flag $F(d)$ (considered over $R_{f}$ ). So after passing to a localization $R_{f}$ of $R$ and choosing a basis of the free $R_{f}$-module $\mathcal{M}$, we can assume $\mathcal{M} \otimes R_{f}$ is an element in $\mathcal{R}\left(\mathcal{F}_{t, l}(\Delta) ; d\right)\left(R_{f}\right)$. If, in particular, $R$ is a local ring (for example the ring of dual numbers), then isomorphism classes of families are in one-to-one correspondence with orbits in $\mathcal{R}\left(\mathcal{F}_{t, l}(\Delta) ; d\right)(R)$ under the action of $\operatorname{GL}(\Sigma d)(R)$.

Let $x$ be an element in $\mathfrak{p}_{u}^{(l)}(d)$ and consider a family $\bar{x}$ in $\mathfrak{p}_{u}^{(l)}(d) \otimes k[\varepsilon] / \varepsilon^{2}$ with $\bar{x} \otimes_{k[\varepsilon] / \varepsilon^{2}} k=x(\varepsilon)=x$, then $\bar{x}$ is just a tangent vector in $x$, so an element in $T_{x}\left(\mathfrak{p}_{u}^{(l)}(d)\right)$. Then the group $\mathrm{GL}(\Sigma d)\left(k[\varepsilon] / \varepsilon^{2}\right)$ acts on the space of all families $\mathcal{F}$ of elements in $\mathfrak{p}_{u}^{(l)}(d)$ over the ring $k[\varepsilon] / \varepsilon^{2}$. The orbit under this action intersected with $T_{x}\left(\mathfrak{p}_{u}^{(l)}(d)\right)$ can be naturally be identified with the quotient $T_{x}\left(\mathfrak{p}_{u}^{(l)}(d)\right) / T_{x}(P \cdot x)$. We can consider the analogous families of $\Delta$-good $\mathcal{A}_{t, l}-$ modules. Then the main step in the proof of Theorem 3.1 consists of the identification of the respective quotients of the tangent spaces.

The following result shows in particular that for a family of $\Delta$-good $\mathcal{A}_{t, l^{-}}$ modules we can at least on a sufficiently small Zariski open subset construct a family of elements in $\mathfrak{p}_{u}^{(l)}(d)$. Moreover, for $x \in \mathfrak{p}_{u}^{(l)}(d)$ the codimension of the $P$-orbit of $x$ in $\mathfrak{p}_{u}^{(l)}(d)$ coincides with the dimension of the extension group $\operatorname{Ext}^{1}(M(x), M(x))$. This result was first proven in Hl]. The Artin-Voigt Lemma (see [Gb, §1]) tells us that $\operatorname{dim} \operatorname{Ext}^{1}(M(x), M(x))$ coincides with the dimension of the quotient of the scheme-theoretic tangent space of the representation space of all $\mathcal{A}_{t, l}-$ modules in $M(x)$ by the tangent space along the GL $(\Sigma d)$-orbit. Since 
$\mathcal{R}\left(\mathcal{F}_{t, l}(\Delta) ; d\right)$ is an open subscheme we can, for the $\Delta$-good module $M(x)$, also consider the dimension of $T_{M(x)}\left(\mathcal{R}\left(\mathcal{F}_{t, l}(\Delta)\right) ; d\right) / T_{M(x)}(\mathrm{GL}(\Sigma d) \cdot M(x))$.

Theorem 3.1. Let $x$ be an element in $\mathfrak{p}_{u}^{(l)}(d)$ and $M(x)$ the corresponding $\Delta$ good $\mathcal{A}_{t, l}-$ module. Then the codimension of the orbit $P \cdot x$ in $\mathfrak{p}_{u}^{(l)}(d)$ coincides with the dimension of $\operatorname{Ext}^{1}(M(x), M(x))$. In particular, the element $x$ is a representative of a dense orbit precisely when $\operatorname{Ext}^{1}(M(x), M(x))=0$.

Proof. We consider an affine family $\mathcal{F}$ of elements in $\mathfrak{p}_{u}^{(l)}(d)$ : this is just an element in $\mathfrak{p}_{u}^{(l)}(d) \otimes R$ for an affine $k$-algebra $R$. To prove the theorem we only require the case where $R$ is the ring of dual numbers $k[\varepsilon] / \varepsilon^{2}$; however the arguments apply for any affine $k$-algebra $R$. Such an affine family $\mathcal{F}$ defines an $\mathcal{A}_{t, l}$-module $\mathcal{M}$, free over $R$, as follows: for $\phi_{i}$ we can take the inclusion $V_{i} \rightarrow V_{i+1}$ (and extend it to $V_{i} \otimes R \subseteq V_{i+1} \otimes R$ ) and we define $\psi_{i}=$ $\left.\mathcal{F}\right|_{V_{i+1+l} \otimes_{k} R} \in \operatorname{Hom}\left(V_{i+1+l}, V_{i}\right) \otimes R$. So it is an element in $\left(\bigoplus \operatorname{Hom}\left(V_{i}, V_{i+1}\right) \oplus\right.$ $\left.\bigoplus \operatorname{Hom}\left(V_{j+l+1}, V_{j}\right)\right) \otimes R$ satisfying the same conditions (relations and injectivity) like a $\Delta$-good representation of $\mathcal{A}_{t, l}$. Moreover, the maps $\phi_{i}$ are obviously locally direct (they are in fact split over $R$ ). In this way each affine family of elements in $\mathfrak{p}_{u}^{(l)}(d)$ defines a corresponding family of $\mathcal{A}_{t, l}$-modules.

Conversely, we also need to show that for each affine family $\mathcal{M}$ of $\mathcal{A}_{t, l^{-}}$ modules, we can cover $\mathcal{M}$ by families $\mathcal{N}$ for which there is a corresponding family $\mathcal{F}$ of elements in $\mathfrak{p}_{u}^{(l)}(d)$. We cannot expect to get a corresponding family of elements in $\mathfrak{p}_{u}^{(l)}(d)$ for all affine families of $\mathcal{A}_{t, l}-$ modules.

In order to see this we consider the affine space

$$
\mathcal{R}\left(\mathbb{A}_{t} ; d\right)=\left\{\left(\phi_{i}\right) \in \bigoplus_{i=1}^{t-1} \operatorname{Hom}\left(V_{i}, V_{i+1}\right) \mid \operatorname{dim} V_{i}=(\Sigma d)_{i}\right\}
$$

of representations of a directed quiver of type $\mathbb{A}_{t}$. It contains the open subscheme

$$
\mathcal{R}\left(\mathbb{A}_{t} ; d\right)^{\mathrm{inj}}=\left\{\left(\phi_{i}\right) \in \bigoplus_{i=1}^{t-1} \operatorname{Hom}\left(V_{i}, V_{i+1}\right) \mid \operatorname{dim} V_{i}=(\Sigma d)_{i}, \phi_{i} \text { is injective }\right\} .
$$

and there is a natural projection morphism

$$
\pi: \mathcal{R}\left(\mathcal{F}_{t, l}(\Delta) ; d\right) \longrightarrow \mathcal{R}\left(\mathbb{A}_{t} ; d\right)^{\mathrm{inj}}, \quad\left(\phi_{i}, \psi_{j}\right) \mapsto\left(\phi_{i}\right) .
$$

Then the $R$-valued points $\mathcal{R}\left(\mathbb{A}_{t} ; d\right)^{\mathrm{inj}}(R)$ consist of split injective maps $\phi=\left(\phi_{i}\right)$. In particular, we can find an element $g \in \mathrm{GL}(\Sigma d)(R)$ so that $g \cdot \phi$ corresponds to the standard flag. Now let $\mathcal{M}$ be a family of $\mathcal{A}_{t, l}$-modules. By passing to a certain localization of $R$, we may assume $\mathcal{M}$ is an element in $\mathcal{R}\left(\mathcal{F}_{t, l}(\Delta) ; d\right)(R)$. We choose an element $g$, so that $g \cdot \pi(\mathcal{M})$ is the standard flag $F(d)$ and consider the family $g \cdot \mathcal{M}$ equivalent to $\mathcal{M}$. Using the definition of the scheme $\mathcal{R}\left(\mathcal{F}_{t, l}(\Delta) ; d\right)$, the element $\psi_{t-l-1}$ for $g \cdot \mathcal{M}$ is an element in $\mathfrak{p}_{u}^{(l)}(d) \otimes R$, the family we want to construct. 
Note that a family over the ring of dual numbers is just a tangent vector, both for $\mathfrak{p}_{u}^{(l)}(d)$ and $\mathcal{R}\left(\mathcal{F}_{t, l}(\Delta), d\right)$, and two families are equivalent precisely if the corresponding tangent vectors are in the same orbit under the group action. Both constructions above preserve the group action and the equivalence classes of the families.

Thus we obtain the first isomorphism below; the second isomorphism is given by the Artin-Voigt lemma.

$$
\begin{aligned}
T_{x}\left(\mathfrak{p}_{u}^{(l)}(d)\right) / T_{x}(P(d) \cdot x) & \cong T_{M(x)}\left(\mathcal{R}\left(\mathcal{F}_{t, l}(\Delta) ; d\right)\right) / T_{M(x)}(\mathrm{GL}(\Sigma d) \cdot(M(x))) \\
& \cong \operatorname{Ext}^{1}(M(x), M(x))
\end{aligned}
$$

Here we consider the Zariski tangent space $T_{x}\left(\mathfrak{p}_{u}^{(l)}(d)\right)$ of $\mathfrak{p}_{u}^{(l)}(d)$ in $x$, the Zariski tangent space $T_{x}(P(d) \cdot x)$ of the $P$-orbit of $x$ in $x$, the Zariski tangent space $T_{M(x)}\left(\mathcal{R}\left(\mathcal{F}_{t, l}(\Delta) ; d\right)\right)$ of $\mathcal{R}\left(\mathcal{F}_{t, l}(\Delta), d\right)$ in $M(x)$, and the Zariski tangent space of the orbit of $M(x)$. This orbit consists precisely of the representations isomorphic to $M(x)$.

In particular, the proof shows that the scheme $\mathcal{R}\left(\mathcal{F}_{t, l}(\Delta), d\right)$ is reduced. Let $\mathcal{M}$ be a family of modules over $\mathcal{A}_{t, l}$ over $k[\varepsilon] / \varepsilon^{2}$. If the projection of the tangent direction of this family to the flag variety $\mathcal{R}\left(\mathbb{A}_{t} ; d\right)^{\mathrm{inj}}$ is not zero, we can find a new equivalent family $\overline{\mathcal{M}}$ having this property. Then this gives a family of elements in $\mathfrak{p}_{u}^{(l)}(d)$, which we can extend it to a family over $R=k[T]$, since $\mathfrak{p}_{u}^{(l)}(d)$ is an affine space. Going back we obtain a family of $\mathcal{A}_{t, l}$-modules equivalent to the original family $\mathcal{M}$. So we can also extend $\mathcal{M}$ to a family over $R=k[T]$ and $\mathcal{R}\left(\mathcal{F}_{t, l}(\Delta), d\right)$ is reduced. This result is a little surprising, since the scheme of all representations of $\mathcal{A}_{t, l}$ is in general not reduced as follows from GS, Thm. 6.6]. If the maps $\phi_{i}$ are not assumed to be injective, we cannot prove such a result.

Finally we note, that in the sequel, we only require one implication of the theorem and apply it for $l=1$; that is we only use that the codimension of $P \cdot x$ in $\mathfrak{p}_{u}^{(1)}$ is at most the dimension of $\operatorname{Ext}^{1}(M(x), M(x))$. In our case, using a certain filtration, it is possible to obtain upper bounds for the dimension of the extension groups, whereas it seems difficult to obtain lower bounds.

\section{Action with a dense orbit}

In this section we prove the direction of Theorem 1.1(1) giving existence of a dense $B$-orbit in $\mathfrak{n}$ in the stated cases.

Let $d$ be a thin dimension vector. Let $B$ be the corresponding Borel subgroup of $\mathrm{GL}_{n}(k)$ and $\mathfrak{n}=\mathfrak{p}_{u}^{(1)}(d)$ the corresponding ideal of $\mathfrak{b}$, see Notation 2.3. We associate to $d$ the sequence $\underline{a}=\underline{a}(d)=\left(a_{0}, a_{1}, \ldots, a_{r}, a_{r+1}\right)$ and set $e(d)=\sharp\{i \mid$ $1 \leq i \leq r, a_{i}$ is even $\}$ as in Definition 2.1. We prove that if $e(d)$ at most one, then $\mathfrak{n}$ is a prehomogeneous space for $B$. In the next section we construct an explicit representative $x$ of the dense orbit. 
It is not feasible to prove that $B$ acts on $\mathfrak{n}$ with a dense orbit using purely algebraic group theoretic results; such an argument would be very technical for the case $e(d)=1$. Instead we show that a certain $\Delta$-good module $M$ with $\Delta$ dimension vector $d$ has no self-extensions. Consequently, there is dense $B$-orbit in $\mathfrak{n}$ by Theorem 3.1

First we consider the case $e(d)=0$. In this case we decompose $d=d^{1}+d^{2}$ where

$$
d_{i}^{1}=\left\{\begin{array}{cl}
d_{i} & \text { if } i \text { is odd } \\
0 & \text { if } i \text { is even }
\end{array}\right.
$$

and

$$
d_{i}^{2}=\left\{\begin{array}{cl}
d_{i} & \text { if } i \text { is even } \\
0 & \text { if } i \text { is odd }
\end{array}\right.
$$

Let $J$ and $K$ be the supports of $d^{1}$ and $d^{2}$ respectively. We have corresponding standard modules $\Delta(J)$ and $\Delta(K)$ and the condition $e(d)=0$ implies that either $\Delta(J) \in\{P(t-1), P(t)\}$ or $\Delta(K) \in\{P(t-1), P(t)\}$. Therefore, by parts (2) and (3) of Theorem 2.4 we have $\operatorname{Ext}^{1}(\Delta(J), \Delta(J))=\operatorname{Ext}^{1}(\Delta(J), \Delta(K))=$ $\operatorname{Ext}^{1}(\Delta(K), \Delta(J))=\operatorname{Ext}^{1}(\Delta(K), \Delta(K))=0$. Hence, setting $M=\Delta(J) \oplus$ $\Delta(K)$ we have $\operatorname{dim}_{\Delta} M=d$ and $\operatorname{Ext}^{1}(M, M)=0$. Therefore, $\mathfrak{n}$ is a prehomogeneous space for $B$ by Theorem 3.1

Now consider the case $e(d)=1$, so $\underline{a}$ has one internal even entry. The construction of a $\Delta$-good module $M$ with $\operatorname{dim}_{\Delta}(M)=d$ and no self-extensions is more complicated in this case. The idea is to construct $M$ as an extension of two certain standard modules $S$ and $T$. Using homological algebra we show, that there exists a unique non-trivial extension

$$
0 \longrightarrow S \longrightarrow M \longrightarrow T \longrightarrow 0
$$

as an $\mathcal{A}_{t, 1}$-module and this module $M$ satisfies $\operatorname{Ext}^{1}(M, M)=0$ (Theorem4.2).

We start with the construction of the standard modules $T=\Delta(J)$ and $S=\Delta(K)$. Let $b$ be the position of the end of the internal even 1-string in $d$ and set

$$
J=\{\ldots, b-5, b-3, b-1, b+2, b+4, b+6, \ldots\} .
$$

Now define $K$ to be disjoint from $J$ and such that $J \cup K$ is the support of $d$. We let $c \in\{t-1, t\}$ be the greatest element of $J$.

Example 4.1. Let $t=18$ and let $d=(1,1,0,1,0,1,0,1,1,1,0,1,1,1,1,0,1,1)$. Then $b=15, J=\{2,4,6,8,10,12,14,17\}, K=\{1,9,13,15,18\}$ and $c=17$.

We are now in a position to state the main theorem of this section.

Theorem 4.2. (a) $\operatorname{Ext}^{1}(T, S)=k$ and $\operatorname{Ext}^{1}(S, T)=0$

(b) If $M$ is the unique non-trivial extension $0 \longrightarrow S \longrightarrow M \longrightarrow T \longrightarrow 0$, then $\operatorname{Ext}^{1}(M, M)=0$.

This theorem along with Theorem 3.1 implies that $B$ acts on $\mathfrak{n}$ with a dense orbit. We prove the theorem in several steps starting with an explicit projective resolution in Lemma 4.3] and proceed with some vanishing results. 
In the next lemma we use the notation for morphisms between standard modules given in Definition 2.6

Lemma 4.3. $0 \longrightarrow P(b) \stackrel{\psi}{\longrightarrow} P(b-1) \oplus P(c) \longrightarrow 0$ is a projective resolution of $T$, where $\psi=\left(\phi^{b-1}, \phi^{b}\right)$.

Proof. Since $T$ is a standard module, it is easy to obtain the result using the covering of $T$ and the covering of the projective modules as explained in BH1]. Alternatively one can prove the result using the explicit description of the projective modules and the morphisms between them given in Section 2

Proof of Theorem 4.2. We first prove part (a). We have $\operatorname{Ext}^{1}(S, T)=0$ by Lemma 2.8 So we are left to show that $\operatorname{Ext}^{1}(T, S)=k$.

Applying $\operatorname{Hom}(-, S)$ to the projective resolution given in Lemma 4.3, we obtain the long exact sequence

$$
\begin{aligned}
0 \longrightarrow \operatorname{Hom}(T, S) \longrightarrow \operatorname{Hom}(P(b-1), S) \oplus \operatorname{Hom}(P(c), S) \longrightarrow & \\
\longrightarrow & \operatorname{Hom}(P(b), S) \longrightarrow \operatorname{Ext}^{1}(T, S) \longrightarrow 0 .
\end{aligned}
$$

Let $l=\sharp(K \cap\{1, \ldots, b-1\})$ and $m=\sharp(K \cap\{1, \ldots, c\})$. The dimensions of Hom-groups given below can be determined using Lemma 2.5 .

$$
\begin{gathered}
\operatorname{hom}(T, S)=m, \operatorname{hom}(P(b-1), S)=l, \operatorname{hom}(P(c), S)=m, \\
\text { and } \operatorname{hom}(P(b), S)=l+1 .
\end{gathered}
$$

Consequently, $\operatorname{ext}^{1}(T, S)=1$.

b) We show $\operatorname{Ext}^{1}(M, M)=0$ in several steps using the exact sequence (4.1) including $M$. First we note that by Theorem 2.4(3), we have $\operatorname{Ext}^{1}(T, T)=0=$ $\operatorname{Ext}^{1}(S, S)$.

Claim 1. $\operatorname{Ext}^{1}(M, S)=0$ :

Applying $\operatorname{Hom}(-, S)$ to (4.1) we obtain a long exact sequence containing

$$
\operatorname{Hom}(S, S) \longrightarrow \operatorname{Ext}^{1}(T, S) \longrightarrow \operatorname{Ext}^{1}(M, S) \longrightarrow \operatorname{Ext}^{1}(S, S)=0 .
$$

Since the extension including $M$ is non-trivial, the first map in the sequence above is not zero. Therefore, it is surjective, which implies that $\operatorname{Ext}^{1}(M, S) \cong$ $\operatorname{Ext}^{1}(S, S)=0$.

Claim 2. $\operatorname{Ext}^{1}(M, T)=0$ :

Applying $\operatorname{Ext}^{1}(-, T)$ to (4.1) we obtain an exact sequence

$$
0=\operatorname{Ext}^{1}(T, T) \longrightarrow \operatorname{Ext}^{1}(M, T) \longrightarrow \operatorname{Ext}^{1}(S, T)=0 .
$$

Claim 3. $\operatorname{Ext}^{1}(M, M)=0$ :

Finally we have the exact sequence

$$
0=\operatorname{Ext}^{1}(M, S) \longrightarrow \operatorname{Ext}^{1}(M, M) \longrightarrow \operatorname{Ext}^{1}(M, T)=0,
$$

which is obtained by applying $\operatorname{Hom}(M,-)$ to 4.1). Hence, $\operatorname{Ext}^{1}(M, M)=0$ as required. 
Remark 4.4. Fix the standard subset $J$ of $\{1, \ldots, t\}$ as above, let $K$ be any standard subset. Let $T=\Delta(J)$ and $S=\Delta(K)$. We note that the key properties of the standard subset $K$ for the proof of Theorem 4.2 are $\operatorname{Ext}^{1}(S, T)=0$ and $b \in K$. There are additional possibilities for $S$ such that $\operatorname{Ext}^{1}(S, T)=0$ provided by Lemma 2.8 Therefore, the same arguments can be used to prove a more general result that $P(d)$ acts on $\mathfrak{p}_{u}^{(1)}(d)$ with a give a dense orbit, when $d$ may also contain some entries 2 .

\section{Representatives of the dense orbits}

In this section we construct a representative of the dense orbit of $B$ in $\mathfrak{n}$ for the cases where such an orbit exists. In Section 6 we use these explicit constructions to prove that a dense $B$-orbit does not exist in the cases given by Theorem 1.1(1). The construction is similar to that given in [BHRR, §8] for Richardson elements in parabolic subgroups of $\mathrm{GL}_{n}(k)$.

Let $d$ be a thin dimension vector with at most one internal even 1-string. Let $B$ be the corresponding Borel subgroup of $\mathrm{GL}_{n}(k)$ and $\mathfrak{n}$ the corresponding ideal of $\mathfrak{b}$, see Notation 2.3 We also use the relabeled basis $\left\{f_{j} \mid j \in \operatorname{supp} d\right\}$ defined in Notation 2.3 and the elementary matrices $f_{i j}$ with respect to this basis.

Below we construct two representatives of the dense $B$-orbit in $\mathfrak{n}$. For the first representative $x$ it is easy to see the module structure of the corresponding $\mathcal{A}_{t, 1^{-}}$module $M(x)$. We prove that the second representative $\bar{x}$ is minimal in the sense of Gd2] Defn. 7.1], this minimality in important for the proofs in the subsequent sections.

We consider the case where $d$ has one internal even 1-string. At the end of this section (Remark [5.5), we explain how to simplify the construction in order to get a representative of the dense $B$-orbit in the easier case when $d$ has no even internal 1-strings.

Let $b$ be the position of the end of the even 1-string in $d$ and decompose the support of $d$ into standard subsets $J$ and $K$ as in the previous section.

The construction of $x$ begins by defining a diagram $D(d)$ in the plane.

1. Place vertices at the coordinates $(j, 1)$ for $j \in J$ and $(i, 0)$ for $i \in K$.

2. From each vertex which is not leftmost in its row draw an arrow to the vertex to its left.

3. Draw an arrow from the vertex at the point $(b+2,1)$ to the vertex at $(b, 0)$.

4. Label the vertices by their $x$-coordinates.

5. Let $x$ be the matrix which is the sum of the elementary matrices $f_{i j}$ for which there is an arrow from vertex $j$ to vertex $i$. 
We note that the Jordan normal form of the nilpotent matrix $x$ as defined above consists of two Jordan blocks of size $\sharp J$ and $\sharp K$.

We illustrate the construction of $x \in \mathfrak{n}$ in an example.

Example 5.1. Let $d=(1,1,0,1,0,1,1,0,1,1,1)$, so $\underline{a}=(2,1,2,3)$. Then we have $b=7, J=\{2,4,6,9,11\}$ and $K=\{1,7,10\}$ and we construct the diagram

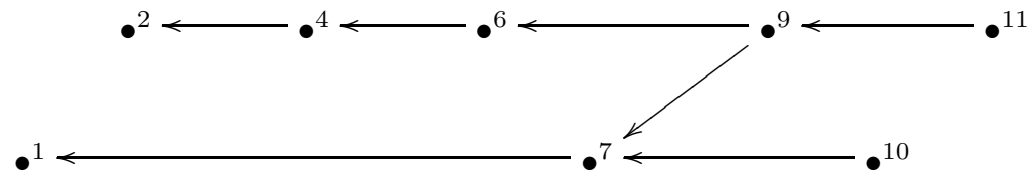

This gives the representative

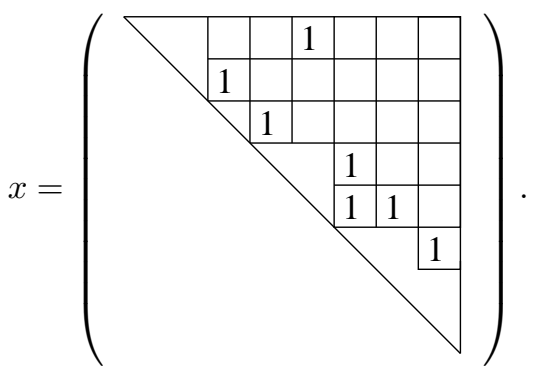

To construct our second representative $\bar{x}$ we have to slightly modify the diagram defining $x$ to obtain a diagram $\bar{D}(d)$. If there is no vertex at a point $(i, 0)$ with $i>b$, then no modification to $D(d)$ is required. Otherwise let $i$ be minimal such that $i>b$ and there is a vertex at $(i, 0)$ in $D(d)$; then we replace the arrow from vertex $i$ to vertex $b$ by an arrow from $i$ to $b-1$. Now $\bar{x}$ is defined from $\bar{D}(d)$ in the same way that $x$ is defined from $D(d)$.

Now we show that $x$ and $\bar{x}$ are conjugate under $B$. We may assume that there is a vertex at a point $(i, 0)$ with $i>b$, and we let $i_{1}<\cdots<i_{s}$ be all such $i$. By construction of $J$ and $K$ there must be at least $s$ vertices with $y$-coordinate 1 that lie to the right of the vertex $b+2$. Let $b+2=j_{1}<\cdots<j_{s}$ be the labels of the first $s$ such vertices. Consider the change of base of $k^{n}$ given by $f_{i_{l}} \mapsto f_{j_{l}}-f_{i_{l}}$, for $l=1, \ldots, s$. It is clear that the matrix $g$ corresponding to this change of base lies in $B$ and that $g x g^{-1}=\bar{x}$.

Example 5.2. We continue with $d$ as in Example 5.1 Then $\bar{D}(d)$ is as below

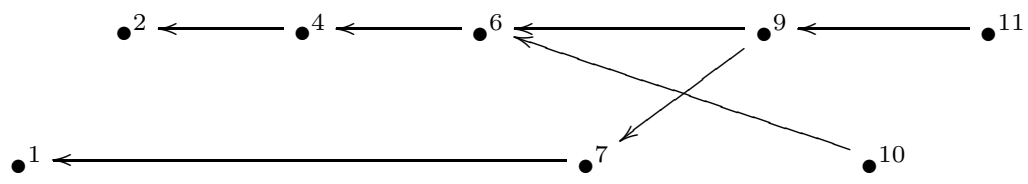


and determines the representative

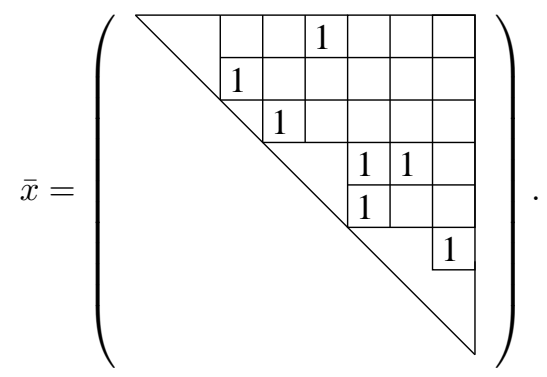

The matrix conjugating $x$ to $\bar{x}$ is

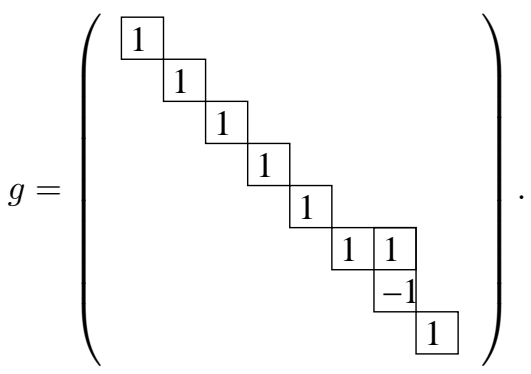

Let $x, \bar{x} \in \mathfrak{n}$ be constructed from the diagrams $D(d)$ and $\bar{D}(d)$ respectively. In Proposition 5.4 below we prove that $x$ and $\bar{x}$ are representatives of the dense $B$-orbit in $\mathfrak{n}$. Further, we prove that, with respect to a certain enumeration of the positive roots of the root system of $\mathrm{GL}_{n}(k)$, we have that $\bar{x}$ is the minimal representative of its $B$-orbit in the sense of [Gd2, Defn. 7.1], see also VA]. In particular, this means that $\bar{x}$ (and $x$ ) have minimal number of nonzero entries. In order to achieve this, we need to give some notation and recall some terminology from Gd2. We warn the reader that in order to simplify our proofs in the sequel some of the notation we give below is nonstandard. In particular, our labelling of the roots of $\mathrm{GL}_{n}(k)$ uses the relabeled basis $\left\{f_{j} \mid j \in \operatorname{supp} d\right\}$ of $k^{n}$.

Let $T$ be the maximal torus of $\mathrm{GL}_{n}(k)$ consisting of diagonal matrices. Let $\Phi=\{(i, j) \in \operatorname{supp} d \times \operatorname{supp} d \mid i \neq j\}$ be the root system of $\mathrm{GL}_{n}(k)$ with respect to $T$, and let $\Phi^{+}=\{(i, j) \in \Phi \mid i<j\}$ be the system of positive roots determined by $B$. For a root $\beta \in \Phi^{+}$we write $\mathfrak{g}_{\beta}$ for the corresponding root subspace of $\mathfrak{u}$. We define $\Phi(\mathfrak{n})=\left\{\beta \in \Phi^{+} \mid \mathfrak{g}_{\beta} \subseteq \mathfrak{n}\right\}$ to be the set of roots of $\mathfrak{n}$ with respect to $T$.

We define a linear order $\sqsubset$ on $\Phi^{+}$by $(i, j) \sqsubset\left(i^{\prime}, j^{\prime}\right)$ if $j<j^{\prime}$, or $j=j^{\prime}$ and $i>i^{\prime}$. Then we enumerate $\Phi(\mathfrak{n})=\left\{\beta_{1}, \ldots, \beta_{\operatorname{dim} \mathfrak{n}}\right\}$ so that $\beta_{l} \sqsubset \beta_{m}$ if and only if $l<m$; we write $\beta_{l}=\left(i_{l}, j_{l}\right)$. For example, if $\mathfrak{n}=\mathfrak{u}$ and $d=(1,1,0,1)$, then we have $\beta_{1}=(1,2), \beta_{2}=(2,4), \beta_{3}=(1,4), \beta_{4}=(2,4)$. We define $B$-submodules $\mathfrak{m}_{l}$ of $\mathfrak{n}$ by $\mathfrak{m}_{l}=\bigoplus_{m=l+1}^{\operatorname{dim} \mathfrak{n}} \mathfrak{g}_{\beta_{m}}$ for $l=0, \ldots, \operatorname{dim} \mathfrak{n}$. Then we define $\mathfrak{n}_{l}=\mathfrak{n} / \mathfrak{m}_{l}$.

We study the orbits of $B$ in $\mathfrak{n}$ by considering the action of $B$ on successive $\mathfrak{n}_{l}$. Let $y \in \mathfrak{n}$ and let $\pi_{l}: \mathfrak{n}_{l} \rightarrow \mathfrak{n}_{l-1}$ be the natural projection. Consider the 
fibre

$$
\pi_{l}^{-1}\left(y+\mathfrak{n}_{l-1}\right)=\left\{y+\lambda e_{i_{l}, j_{l}}+\mathfrak{m}_{l}: \lambda \in k\right\} \subseteq \mathfrak{n}_{l} .
$$

Then [Gd2, Lem. 5.1] (see also [VA Lem. 3.1]) says that for $y \in \mathfrak{n}$ either

(I) all elements of $\pi_{l}^{-1}\left(y+\mathfrak{n}_{l-1}\right)$ are $U$-conjugate or

(R) no two elements of $\pi_{l}^{-1}\left(y+\mathfrak{n}_{l-1}\right)$ are $U$-conjugate.

We say that $l$ is an inert point of $y$ if (I) holds; and we say that $l$ is a ramification point of $y$ if (R) holds.

In Gd2, Defn. 7.1] minimal representatives of $B$-orbits in $\mathfrak{n}_{l}$ (with respect to an enumeration of $\Phi^{+}$) are defined. We recall that Gd2 Prop. 7.2 and Rem. $7.3]$ says that a $B$-orbit in $\mathfrak{n}_{l}$ contains a unique minimal representative. Further, Gd2, Lem. 5.5] says that $y=\sum_{m=1}^{l} a_{m} e_{i_{m}, j_{m}}+\mathfrak{m}_{l}$ is the minimal representative of its $B$-orbit in $\mathfrak{u}_{l}$ if and only if: $a_{m}=0$ whenever $m$ is an inert point of $y$; and if $a_{m} \neq 0$ and $\beta_{m}$ is linearly independent of $\left\{\beta_{m^{\prime}} \mid m^{\prime}<m\right.$ and $\left.a_{m^{\prime}} \neq 0\right\}$, then $a_{m}=1$. From the above description of minimal representatives of $B$-orbits in $\mathfrak{n}_{l}$, it follows that if $y+\mathfrak{m}_{l}$ is the minimal representative of its $B$-orbit in $\mathfrak{n}_{l}$ and $l^{\prime}<l$, then $y+\mathfrak{m}_{l^{\prime}}$ is the minimal representative of its $B$-orbit in $\mathfrak{n}_{l^{\prime}}$.

The convention discussed in the following remark is required in the proofs of both Propositions 5.4 and 6.1

Remark 5.3. Let $m \neq t$. By definition of the function $\gamma$ in Notation $2.3 \mathrm{~m}$ is the $\gamma(m)$ th lowest element of $\operatorname{supp} d$. We define $h_{m} \in\{1, \ldots, M\}$ to be minimal so that $j_{h_{m}+1}>m$. Let $B_{\gamma(m)}$ be the Borel subgroup of upper triangular matrices in $\operatorname{GL}_{\gamma(m)}(k)$, then we can identify $\mathfrak{n}_{h_{m}}$ with an ideal in the Lie algebra $\mathfrak{b}_{\gamma(m)}$ of $B_{\gamma(m)}$. Under this identification we see that two elements of $\mathfrak{n}_{h_{m}}$ are $B$ conjugate if and only if they are $B_{\gamma(m)}$-conjugate. Also the identification allows us to consider Jordan normal forms of elements of $\mathfrak{n}_{h_{m}}$.

We can now state the main proposition of this section.

Proposition 5.4. Let $x, \bar{x} \in \mathfrak{n}$ be constructed from the diagrams $D(d)$ and $\bar{D}(d)$ respectively. Then $x$ and $\bar{x}$ are representatives of the dense $B$-orbit in $\mathfrak{n}$. Moreover, $\bar{x}$ is the minimal representative of this B-orbit.

Proof. Consider the $\mathcal{A}_{t, 1}$-module $M(x)$ corresponding to $x$ as defined in Section 4. We see that the subspace of $k^{n}$ spanned by $\left\{f_{i}: i \in K\right\}$ is stable under the action of $x$. It follows that there is a submodule $S$ of $M(x)$ isomorphic to $\Delta(K)$. Further, we see that the quotient $T=M(x) / S$ is isomorphic to $\Delta(J)$. Now consider the direct sum of $T \oplus S$. We can see $T \oplus S=M(y)$, where $y=x-f_{b, b+2}$. Now it is clear that $x$ is not $B$-conjugate to $y$, so $M(x)$ is not isomorphic to $T \oplus S$. Therefore, $x$ is a representative of the dense $B$-orbit in $\mathfrak{n}$ by Theorems 3.1 and 4.2 Since $\bar{x}$ is in the same $B$-orbit as $x$ it follows that $\bar{x}$ is also a representative of the dense $B$-orbit.

We define $A$ to be the subset of $\{1, \ldots, \operatorname{dim} \mathfrak{n}\}$ such that $\bar{x}=\sum_{l \in A} f_{i_{l}, j_{l}}$. To show that $\bar{x}$ is the minimal representative in its $B$-orbit it suffices to show that each $l \in A$ is a ramification point of $\bar{x}$. 
Let $l \in A$. If $i_{l}=j_{l}-2$, then it is clear that $l$ is a ramification point of $x$, so assume that $l$ is such that $i_{l}<j_{l}-2$. Then the vertex $j_{l}$ has $y$-coordinate 0 , or $j_{l}=b+2$ and $i_{l}=b-1$. Now suppose for a contradiction that $l$ is an inert point of $x$. Then the minimal representative of the $B$-orbit in $\mathfrak{n}_{h_{j_{l}}}$ is of the form

$$
y+\mathfrak{m}_{h_{j_{l}}}=x-f_{i_{l}, j_{l}}+\sum_{i \in \operatorname{supp} d: i<i_{l}} a_{i} f_{i, j_{l}}+\mathfrak{m}_{h_{j_{l}}},
$$

where $a_{i} \in\{0,1\}$.

We may identify $\mathfrak{n}_{h_{j_{l}}}$ with an ideal in $\mathfrak{b}_{\gamma\left(j_{l}\right)}$ as discussed in Remark 5.3 and thus view $x+\mathfrak{m}_{h_{j_{l}}}$ and $y+\mathfrak{m}_{h_{j_{l}}}$ as endomorphisms of $k^{\gamma\left(j_{l}\right)}$. Further, $x+\mathfrak{m}_{h_{j_{l}}}$ and $y+\mathfrak{m}_{h_{j_{l}}}$ stabilize the subspace $k^{\gamma\left(i_{l}\right)-1}$ so induce endomorphisms of $k^{\gamma\left(j_{l}\right)} / k^{\gamma\left(i_{l}\right)-1}$. The kernels of these induced maps have different dimensions: the map induced by $y+\mathfrak{m}_{h_{j_{l}}}$ has kernel of dimension 3, whereas the kernel of the map induced by $x+\mathfrak{m}_{h_{j_{l}}}$ is 2-dimensional. It follows that $x+\mathfrak{m}_{h_{j_{l}}}$ and $y+\mathfrak{m}_{h_{j_{l}}}$ are not $B_{\gamma\left(j_{l}\right)}$-conjugate. Therefore, $x$ and $y$ cannot be $B$-conjugate. This contradiction implies that $l$ is a ramification point of $x$, as required.

Remark 5.5. To end this section we mention how one can adapt the above construction to give representatives of the dense $B$-orbit in $\mathfrak{n}$ in case $d$ has no internal even 1-strings. In this case we decompose the support of $d$ in to subsets $J$ and $K$ as in the previous section. We construct a diagram using the same method as for $D(d)$ except that we omit step 3, then use this diagram to define $x \in \mathfrak{n}$. One can prove that $x$ is a representative in the dense $B$-orbit in $\mathfrak{n}$ and that it is the minimal representative of this orbit using arguments as in the proof of Proposition 5.4

\section{Actions without dense orbit}

In this section we prove non-existence of a dense $B$-orbit in the cases given by Theorem 1.1 (1). The proof requires the explicit constructions of representatives of dense $B$-orbits (when they exist) given in Section 5

Let $d$ be a thin dimension vector. Let $B$ be the corresponding Borel subgroup of $\mathrm{GL}_{n}(k)$ and $\mathfrak{n}$ the corresponding Lie ideal of $\mathfrak{b}$, see Notation 2.3 We define the sequence $\underline{a}=\underline{a}(d)$ and the number $e(d)$ as in Definition 2.1] For the proof of the following proposition we use the terminology for $B$-orbits given in the previous section. In particular, we use the relabeled standard basis $\left\{f_{j} \mid j \in \operatorname{supp} d\right\}$ of $k^{n}$ and we enumerate the roots $\Phi(\mathfrak{n})$ of $\mathfrak{n}$ as before. Further, we define the quotients $\mathfrak{n}_{l}$ of $\mathfrak{n}$ and we have minimal representatives of $B$-orbits in $\mathfrak{n}_{l}$.

Proposition 6.1. Suppose $e(d) \geq 2$. Then $B$ does not act on $\mathfrak{n}$ with a dense orbit.

Proof. Let $\mathfrak{m}$ be an ideal of $\mathfrak{b}$ contained in $\mathfrak{n}$. If $B$ acts on $\mathfrak{n}$ with a dense orbit, then $B$ also acts on $\mathfrak{n} / \mathfrak{m}$ with a dense orbit. It follows that it suffices to consider the case where we have $\underline{a}=\left(1, a_{1}, \ldots, a_{r}, 1\right)$ with $a_{1}$ and $a_{r}$ even and $a_{i}$ odd for $2 \leq i \leq r-1$. 
We have $\gamma(t-2)=n-1$. Define $h_{t-2}$ and $B_{\gamma(t-2)}=B_{n-1}$ as in Remark 5.3 We can identify $\mathfrak{n}_{h_{t-2}}$ with an ideal in $\mathfrak{b}_{n-1}$, it corresponds to the dimension vector $\hat{d}=\left(d_{1}, \ldots, d_{t-1}\right)$ and the 1 -string sequence $\hat{a}=\left(1, a_{1}, \ldots, a_{r}\right)$. We know there exists a dense orbit for the action of $B$ on $\mathfrak{n}_{h_{t-2}}$ and this dense orbit has minimal representative $\bar{x}+\mathfrak{m}_{h_{t-2}}=\sum_{l \in A} f_{i_{l}, j_{l}}+\mathfrak{m}_{h_{t-2}}$ that can be constructed using the diagram $\bar{D}(\hat{d})$ as described in the previous section. The Jordan normal form of $\bar{x}+\mathfrak{m}_{h_{n-1}}$ consists of two Jordan blocks of size $\lambda \geq \mu$, say. It is easy to see from the construction of $\bar{x}+\mathfrak{n}_{h_{n-1}}$ that we must in fact have $\lambda>\mu$.

Suppose there is a dense $B$-orbit in $\mathfrak{n}$. The minimal representative of this orbit is of the form $\bar{x}=\sum_{l \in A} f_{i_{l}, j_{l}}+\sum_{i=1}^{n-1} c_{i} e_{i, n}$, where $c_{i} \in\{0,1\}$. It is clear that we must have that $c_{n-1}=1$, i.e. $h_{n-1}+1$ is a ramification point of $\bar{x}$. It now follows from [Gd2, Lem. 5.7 and Prop. 7.7] that, for $\bar{x}$ to be a representative of a dense $B$-orbit in $\mathfrak{n}$, we must have that $l$ is an inert point of $x$ for $l=h_{n-1}+2, \ldots, M$; so that $c_{i}=0$ for $i=1, \ldots, n-2$. However, we see that the Jordan normal form of $\bar{x}$ has blocks of size $\lambda \geq \mu+1$, and the Jordan decomposition of $\bar{x}+e_{n-2, n}$ is given by the partition $\lambda+1 \geq \mu$. The closure ordering for conjugacy classes of nilpotent matrices is well-known (see for example [He, Thm. 3.10]) and this ordering tells us that $x+e_{n-2, n}$ is not contained in the closure of the $\mathrm{GL}_{n}(k)$-orbit of $\bar{x}$ and so certainly cannot be contained in the closure of the $B$-orbit of $x$. This contradiction implies that there cannot be a dense $B$-orbit in $\mathfrak{n}$.

\section{The codimension of a maximal $B$-orbit}

In this section we improve our results and compute the codimension of a $B$ orbit of maximal dimension in the ideal $\mathfrak{n}$. In fact we construct a dense family of $B$-orbits in $\mathfrak{n}$ of maximal dimension.

Let $d$ be a thin dimension vector. Let $B$ be the corresponding Borel subgroup of $\mathrm{GL}_{n}(k)$ and $\mathfrak{n}=\mathfrak{p}_{u}^{(1)}(d)$ the corresponding Lie ideal of $\mathfrak{b}$, see Notation 2.3. We define the number $e(d)$ as in Definition 2.1. We denote by $\operatorname{cd}(B ; \mathfrak{n})$ the codimension in $\mathfrak{n}$ of a $B$-orbit of maximal dimension.

The main result in this section is the following theorem, which gives part (2) of Theorem 1.1

Theorem 7.1. Let $d$ be a thin dimension vector. Then

$$
\operatorname{cd}(B ; \mathfrak{n})=\max \{0, e(d)-1\} .
$$

For the remainder of this section we assume that $e(d) \geq 2$. The cases $e(d) \leq 1$ in Theorem 7.1 follow from the results of Section 4 We prove the theorem in two steps. Using homological algebra, we compute an upper bound for $\operatorname{cd}(B ; \mathfrak{n})$. That is we show that for certain $\mathcal{A}_{t, 1}$-modules, we have $\operatorname{Ext}^{1}(M, M) \leq e(d)-1$. We complete the proof by constructing an $(e(d)-1)$-dimensional affine family such that the geometric fibres $\bar{X}$ of the family consists of pairwise non-conjugate elements $x \in \mathfrak{n}$, such that for each $x \in \bar{X}$ the corresponding module $M(x)$ is isomorphic to a module $M$ for which we have shown $\operatorname{Ext}^{1}(M, M) \leq e(d)-1$. 
We obtain the upper bound $\operatorname{cd}(B ; \mathfrak{n}) \leq e(d)-1$ from Lemma 7.3 below and Theorem 3.1 First we introduce some notation that is required for the proof of this lemma.

Let $b_{1}<\cdots<b_{e(d)}$ be the ends of the internal even 1-strings in $d$. Define $J=\left\{\ldots, b_{1}-1, b_{1}+2, \ldots, b_{2}-1, b_{2}+2, \ldots, b_{3}-1, b_{3}+2, \ldots\right\}$ to be the largest standard subset containing $b_{i}-1, b_{i}+2$ and all possible $b_{1}-1-2 j$ and $b_{e(d)}+2+2 j$ for $j \geq 0$. Further, let $c$ be the greatest element of $J$ and let $K$ be the subset of $\{1, \ldots, t\}$ such that supp $d$ is the disjoint union of $J$ and $K$. Define the standard modules $T=\Delta(J)$ and $S=\Delta(K)$.

Example 7.2. Let $d=(1,0,1,1,0,1,1,1,1,0,1,1,1,0,1)$. Then we have $J=$ $\{1,3,6,8,11,13,15\}$ and $K=\{4,7,9,12\}$.

Lemma 7.3. (a) $\operatorname{ext}^{1}(T, S)=e(d)$

(b) $\operatorname{ext}^{1}(S, T)=0$

(c) For any non-trivial extension $M$ of $T$ and $S$ we have $\operatorname{ext}^{1}(M, M) \leq e(d)-1$.

Proof. (a) We first consider a projective resolution of $T$

$$
0 \longrightarrow P\left(b_{1}\right) \oplus \ldots \oplus P\left(b_{m}\right) \stackrel{\psi}{\longrightarrow} P\left(b_{1}-1\right) \oplus \ldots \oplus P\left(b_{e(d)}-1\right) \oplus P(c),
$$

where $\psi=\psi_{1} \oplus \ldots \psi_{e(d)}$, with $\psi_{i}=\left(0, \ldots, 0, \phi^{b_{i}-1}, \phi^{b_{i}}, 0, \ldots, 0\right)$ for $i=$ $1, \ldots, e(d)-1$ and $\psi_{e(d)}=\left(0, \ldots, 0, \phi^{b_{e(d)}-1}, \phi^{b_{e(d)}}\right)$, here we use the notation of Definition 2.6. As in the proof of Theorem 4.2 this gives rise to a long exact sequence.

$0 \longrightarrow \operatorname{Hom}(T, S) \longrightarrow \operatorname{Hom}\left(P\left(b_{1}-1\right), S\right) \oplus \cdots \oplus \operatorname{Hom}(P(c), S) \longrightarrow$

$$
\longrightarrow \operatorname{Hom}\left(P\left(b_{1}\right), S\right) \oplus \cdots \oplus \operatorname{Hom}\left(P\left(b_{e(d)}\right), S\right) \longrightarrow \operatorname{Ext}^{1}(T, S) \longrightarrow 0 .
$$

Computing dimensions of Hom-groups using Lemma 2.5 yields

$$
\begin{gathered}
\operatorname{hom}(T, S)=\operatorname{hom}(P(c), S), \text { and } \\
\operatorname{hom}\left(P\left(b_{i}\right), S\right)=\operatorname{hom}\left(P\left(b_{i}-1\right), S\right)+1 \text { for } i=1, \ldots, e(d) .
\end{gathered}
$$

From this we can calculate $\operatorname{ext}^{1}(T, S)=e(d)$.

(b) Using Lemma 2.8 we get $\operatorname{Ext}^{1}(S, T)=0$.

(c) We consider a non-trivial extension

$$
0 \longrightarrow S \longrightarrow M \longrightarrow T \longrightarrow 0 \text {. }
$$

Applying $\operatorname{Hom}(-, S)$ to the short exact sequence above we obtain a long exact sequence containing

$$
\operatorname{Hom}(S, S) \longrightarrow \operatorname{Ext}^{1}(T, S) \longrightarrow \operatorname{Ext}^{1}(M, S) \longrightarrow 0,
$$

since $\operatorname{Ext}^{1}(S, S)=0$ by Theorem 2.4 4). Moreover, since the first map in the exact sequence above is not the zero map, we obtain $\operatorname{ext}^{1}(M, S) \leq e(d)-1$. Applying $\operatorname{Hom}(-, T)$ to (7.1) we obtain the exact sequence

$$
0=\operatorname{Ext}^{1}(T, T) \longrightarrow \operatorname{Ext}^{1}(M, T) \longrightarrow \operatorname{Ext}^{1}(S, T)=0
$$


and deduce that $\operatorname{Ext}^{1}(M, T)=0$. Finally applying $\operatorname{Hom}(M,-)$ to (7.1) we obtain the exact sequence

$$
\operatorname{Ext}^{1}(M, S) \longrightarrow \operatorname{Ext}^{1}(M, M) \longrightarrow \operatorname{Ext}^{1}(M, T)=0
$$

implying that $\operatorname{Ext}^{1}(M, M) \leq e(d)-1$ as required.

We now explain a construction of a family of elements $x \in \mathfrak{n}$, for which $M(x)$ is a nontrivial extension of $S$ by $T$. This construction generalizes the one given in Section 5 and begins by defining a diagram in the plane. We use the relabeled basis $\left\{f_{j} \mid j \in \operatorname{supp} d\right\}$ of $k^{n}$ from Notation 2.3. We denote the diagram in the plane in this construction by $D(d)$.

1. Place vertices at the coordinates $(j, 1)$ for $j \in J$ and $(i, 0)$ for $i \in K$.

2. From each vertex which is not leftmost in its row and is not at $\left(b_{i}+2,1\right)$ for some $i=2, \ldots, e(d)$, draw an arrow to the vertex on its left.

3. For $i=1, \ldots, e(d)$, draw an arrow from the vertex at the point $\left(b_{i}+2,1\right)$ to the vertex at the point $\left(b_{i}, 0\right)$.

4. For $i=2, \ldots, e(d)$ draw a twiddly arrow from the vertex at $\left(b_{i}+2,1\right)$ to the vertex at $\left(b_{i}-1,1\right)$ and label this twiddly arrow $i$.

5 . Number the vertices by their $x$-coordinate.

6. Let $A \subset\{(i, j) \mid i<j\}$ be the set of all pairs such that there is a nontwiddly arrow from $j$ to $i$.

7. Define $\mathcal{F}=\sum_{(i, j) \in A} f_{i j}+\sum_{i=2}^{e(d)} x_{i} f_{b_{i}-1, b_{i}+2} \in \mathfrak{n} \otimes k\left[x_{2}, x_{2}^{-1}, \ldots, x_{m}, x_{m}^{-1}\right]$, where $x_{2}, \ldots, x_{m}$ are indeterminates. Then $\mathcal{F}$ is a family of elements of $\mathfrak{n}$ over the affine algebra $k\left[x_{2}, x_{2}^{-1}, \ldots, x_{m}, x_{m}^{-1}\right]$.

Example 7.4. Let $d=(1,0,1,1,0,1,1,1,1,0,1,1,1,0,1)$ as in Example 7.2 Then the diagram $D(d)$ is given below

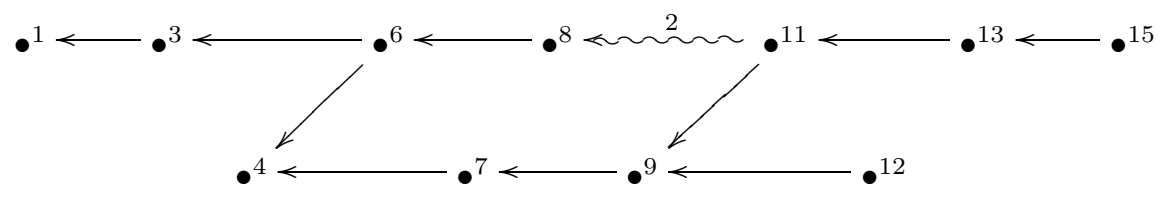

We have

$x=f_{1,3}+f_{3,6}+f_{4,6}+f_{4,7}+f_{6,8}+f_{7,9}+x_{2} f_{8,11}+f_{9,11}+f_{9,12}+f_{11,13}+f_{13,15}$.

Each $\left(t_{2}, \ldots, t_{m}\right) \in\left(k^{\times}\right)^{m}$ defines a maximal ideal of $k\left[x_{2}, x_{2}^{-1}, \ldots, x_{m}, x_{m}^{-1}\right]$, and we write $\mathcal{F}\left(t_{2}, \ldots, t_{m}\right)=\sum_{(i, j) \in A} f_{i j}+\sum_{i=2}^{m} t_{i} f_{b_{i}-1, b_{i}+2} \in \mathfrak{n}$.

The following lemma is proved in the same way as the first part of Proposition 5.4 so we omit the proof. 
Lemma 7.5. Let $T, S$ and $\mathcal{F}$ be as above and let $\left(t_{2}, \ldots, t_{m}\right) \in\left(k^{\times}\right)^{m}$. Then $M\left(\mathcal{F}\left(t_{2}, \ldots, t_{m}\right)\right)$ is a nontrivial extension of $S$ by $T$.

As in Section 5 we require an alternative construction in order to obtain minimal representatives of $B$-orbits. The modification required is explained below.

To construct our second family $\overline{\mathcal{F}}$ we have to slightly modify the diagram $D(d)$ to obtain a diagram $\bar{D}(d)$. For each $i=1, \ldots, e(d)-1$, we let $j_{i}$ be the label of the vertex to the right of $b_{i}$. Replace the arrow from $j_{i}$ to $b_{i}$ by an arrow from $j_{i}$ to $b_{i}-1$. If there is a vertex $j_{e(d)}$ to the right of the vertex $b_{e(d)}$, then we replace the arrow from $j_{e(d)}$ to $b_{e(d)}$ by an arrow from $j_{e(d)}$ to $b_{e(d)}-1$. Now the family $\overline{\mathcal{F}} \in \mathfrak{n} \otimes k\left[x_{2}, x_{2}^{-1}, \ldots, x_{m}, x_{m}^{-1}\right]$ is defined from $\bar{D}(d)$ in the same way that $\mathcal{F}$ is defined from $D(d)$.

Let $\left(t_{2}, \ldots, t_{e(d)}\right) \in\left(k^{\times}\right)^{e(d)-1}$. Then it is straightforward to show that $\mathcal{F}\left(t_{2}, \ldots, t_{e(d)}\right)$ is $B$-conjugate to $\overline{\mathcal{F}}\left(t_{2}, \ldots, t_{e(d)}\right)$. This can be done inductively using the arguments from Section [5 showing that, in the situation of that section, the alternative representatives $x$ and $\bar{x}$ of the dense $B$-orbit are conjugate.

Example 7.6. Let $d$ as in Examples 7.2 and 7.4 Then the diagram $\bar{D}(d)$ is

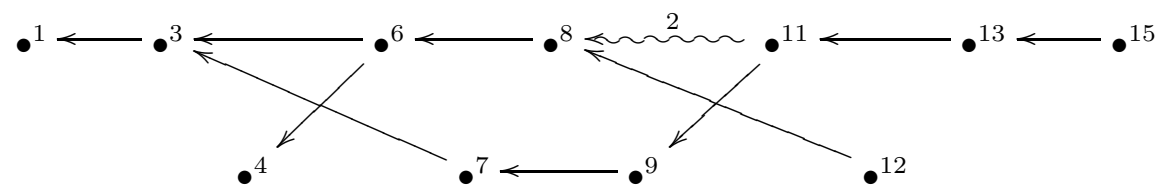

We have

$\overline{\mathcal{F}}=f_{1,3}+f_{3,6}+f_{3,7}+f_{4,6}+f_{6,8}+f_{7,9}+x_{2} f_{8,11}+f_{8,12}+f_{9,11}+f_{11,13}+f_{13,15}$.

For the statement Lemma 7.7 we use the terminology of minimal representatives with the enumeration of $\Phi(\mathfrak{n})$ given in Section 5 The lemma is proved using arguments from the proof of Proposition 5.4 so we omit its proof.

Lemma 7.7. For each $\left(t_{2}, \ldots, t_{e(d)}\right) \in\left(k^{\times}\right)^{e(d)-1}$ have that $\overline{\mathcal{F}}\left(t_{2}, \ldots, t_{e(d)}\right)$ is the minimal representative of its $B$-orbit.

We are now in a position to prove Theorem 7.1

Proof of Theorem 7.1. Let $\bar{X}=\left\{\overline{\mathcal{F}}\left(t_{2}, \ldots, t_{m}\right) \in \mathfrak{n} \mid\left(t_{2}, \ldots, t_{m}\right) \in\left(k^{\times}\right)^{e(d)-1}\right\}$ be the geometric fibres of the family $\overline{\mathcal{F}}$. The elements of $\bar{X}$ are minimal representatives in their $B$-orbits by Lemma 7.7 Therefore, the uniqueness of minimal representatives (Gd2, Prop. 7.2 and Rem. 7.3]) means that no two elements of $\bar{X}$ lie in the same $B$-orbit. By Lemmas 7.3 and 7.5 and Theorem 3.1 the codimension in $\mathfrak{n}$ of the $B$-orbit of any $y \in \mathfrak{n}$ is $e(d)-1$ or less. It follows that $B \cdot \bar{X}=\bigcup_{y \in \bar{X}} B \cdot y$ has dimension dim $\mathfrak{n}$ and is therefore dense in $\mathfrak{n}$. It follows from [Bo, Cor. AG.10.3] that $Z=\left\{z \in \mathfrak{n} \mid \operatorname{codim}_{\mathfrak{n}} B \cdot z<e(d)-1\right\}$ is open in $\mathfrak{n}$. If $Z$ is nonempty, then its intersection with $B \cdot \bar{X}$ is nonempty. Then $Z \cap \bar{X}$ is open and nonempty in $\bar{X}$. But then the dimension of $B \cdot(Z \cap \bar{X})$ is greater than the dimension of $\mathfrak{n}$, which is absurd. Hence, $Z$ is empty and we have $\operatorname{cd}(B ; \mathfrak{n})=e(d)-1$. 
Next we give an example of a dense family of $B$-orbits.

Example 7.8. For $d=(1,0,1,1,1,0,1,0,1,0,1,0,1,1,0,1,0,1,1,1,1,0,1,1)$ we have a family with one parameter $t$. This family is illustrated in Figure 3 below.

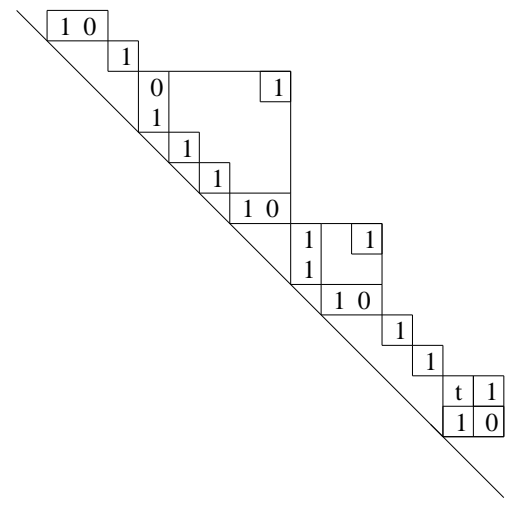

Figure 3: A family of $B$-orbits

\section{Maximal $B(q)$-conjugacy classes in $N(q)$}

We assume, for this section, that $k$ is the algebraic closure of the finite field of $p$ elements $\mathbb{F}_{p}$, where $p$ is a prime. Let $d$ be a thin dimension vector. Let $B$ and $\mathfrak{n}$ be the be the corresponding Borel subgroup of $\mathrm{GL}_{n}(k)$ and Lie ideal of $\mathfrak{b}$, see Notation 2.3. We write $N$ for the unipotent normal subgroup of $B$ with Lie algebra $\mathfrak{n}$.

We note that $B$ has a standard definition over $\mathbb{F}_{p}$ and $N$ is a subgroup of $B$ defined over $\mathbb{F}_{p}$. For a power $q$ of $p$ we write $B(q)$ for the group of $\mathbb{F}_{q}$-rational points in $B$, that is $B(q)=B \cap \mathrm{GL}_{n}(q)$; likewise we write $N(q)$ and $\mathfrak{n}(q)$ for the $\mathbb{F}_{q}$-rational points of $N$ and $\mathfrak{n}$ respectively. The purpose of this final section is to deduce a result (Theorem 8.1) about the maximal size of a $B(q)$-conjugacy class in $N(q)$ from Theorem[7.1] This is achieved using results of the first author from Gd2].

We associate to $d$ the positive integer $e(d)$ as in Definition 2.1 We use the terminology for $B$-orbits recalled from Gd2 in Section 5 In particular, we enumerate $\Phi(\mathfrak{n})$ as in Section 5 then we have minimal representatives of $B$-orbits in $\mathfrak{n}$ and the notion of inert and ramifications points.

Let $y=\sum_{(i, j) \in A} a_{i} f_{i, j} \in \mathfrak{n}(q)$ be the minimal representative of its $B$-orbit in $\mathfrak{n}$, where $A$ is a subset of $\Phi(\mathfrak{n})$ and $a_{i} \in \mathbb{F}_{q}^{\times}$. We write in $(y)$ for the number of inert points of $y$. By [Gd2, Prop. 7.7] we have a factorization $C_{B}(y)=$ $C_{T}(y) C_{U}(y)$ and this induces a factorization $C_{B(q)}(y)=C_{T(q)}(y) C_{U(q)}(y)$. We have that $\operatorname{dim} U \cdot y=\operatorname{in}(y)$ by $\left[\mathrm{Gd2}\right.$, Lem. 5.3] and we have $|U(q) \cdot y|=q^{\text {in }(y)}$ by 
Gd2, Prop. 6.4]. Let $\mathrm{m}(y)$ be the rank of the sublattice of the root lattice of $\Phi$ generated by $A$. Then it is easy to see that $\operatorname{dim} T \cdot y=\mathrm{m}(y)$ and $|T(q) \cdot y|=$ $(q-1)^{\mathrm{m}(y)}$. Putting this all together we have that $\operatorname{dim} B \cdot y=\mathrm{m}(y)+\operatorname{in}(y)$ and $|B(q) \cdot y|=(q-1)^{\mathrm{m}(y)} q^{\operatorname{in}(y)}$.

We are now in a position to prove the theorem of this section.

Theorem 8.1. The maximal size of a $B(q)$-conjugacy class in $N(q)$ is:

(a) $(q-1)^{n-2} q^{\operatorname{dim} N-(n-2)}$, if $e(d)=0$;

(b) $(q-1)^{n-1} q^{\operatorname{dim} N-(n-2+e(d))}$, if $e(d)>0$.

Proof. We first note that the map $x \mapsto 1+x$ from $\mathfrak{n}$ to $N$ is a $B$-equivariant and sends $\mathfrak{n}(q)$ to $N(q)$. Therefore, we can consider the action of $B$ on $\mathfrak{n}$ rather than on $\mathfrak{n}$.

We first consider the case $e(d)=1$. Let $\bar{x}$ be the minimal representative of the dense $B$-orbit in $\mathfrak{n}$ constructed in Section 5 The entries of $\bar{x}$ are all 0 or 1 so we have $\bar{x} \in \mathfrak{n}(q)$. Since $B \cdot \bar{x}$ is dense in $\mathfrak{n}$, we have $\operatorname{dim} B \cdot \bar{x}=\operatorname{dim} \mathfrak{n}$. It is easy to see that $\mathrm{m}(\bar{x})=n-1$ and it follows that $\operatorname{in}(\bar{x})=\operatorname{dim} \mathfrak{n}-(n-1)$. Therefore, we have $|B(q) \cdot \bar{x}|=(q-1)^{n-1} q^{\operatorname{dim} N-(n-1)}$.

Now $B \cdot \bar{x}$ is open and dense $\mathfrak{n}$ and the $U$-orbit of any $z \in B \cdot \bar{x}$ has dimension $\operatorname{dim} \mathfrak{n}-(n-1)$. It follows from Bo, Cor. AG.10.3] that $\operatorname{dim} U \cdot z \leq \operatorname{dim} \mathfrak{n}-(n-1)$ for all $z \in \mathfrak{n}$. It is clear that $\operatorname{dim} T \cdot z \leq n-1$ for any $z \in \mathfrak{n}$. Therefore, if $y \in \mathfrak{n}(q)$ is the minimal representative of its $B$-orbit in $\mathfrak{n}$ we have $\mathrm{m}(y) \leq n-1$ and $\operatorname{in}(y) \leq \mathfrak{n}-(n-1)$, so that $|B(q) \cdot y| \leq(q-1)^{n-1} q^{\operatorname{dim} \mathfrak{n}-(n-1)}$. The case $e(d)=1$ in the theorem now follows, because each $B$-orbit in $\mathfrak{n}$ has a minimal representative.

The case $e(d)=0$ can be proved in more or less the same way. Let $x \in \mathfrak{n}(q)$ be the minimal representative of the dense $B$-orbit as constructed in Section 5 . Then we can show that $|B(q) \cdot x|=(q-1)^{n-2} q^{\operatorname{dim} N-(n-2)}$. In this case it is necessary, to observe that $\mathfrak{n} \backslash(B \cdot x)$ has dimension strictly less than $\operatorname{dim} \mathfrak{n}$. It follows that if $y \in \mathfrak{n} \backslash(B \cdot x)$ is the minimal representative of its $B$-orbit, then $\operatorname{dim} B \cdot y=\mathrm{m}(y)+\operatorname{in}(y)<\operatorname{dim} \mathfrak{n}$, which forces $|B(q) \cdot y|<(q-1)^{n-2} q^{\operatorname{dim} N-(n-2)}$ (an analogous strict inequality could have also been shown in the case $e(d)=1$ ).

The case $e(d)>1$ can be proved in much the same way as for $e(d)=1$. Let $\overline{\mathcal{F}} \in \mathfrak{n} \otimes k\left[x_{2}, x_{2}^{-1}, \ldots, x_{e(d)}, x_{e(d)}^{-1}\right]$ be the family of elements of $\mathfrak{n}$ from Section 7 Then for $\left(t_{2}, \ldots, t_{e(d)}\right) \in \mathbb{F}_{q}^{\times}$, we have that

$$
\left|B(q) \cdot \overline{\mathcal{F}}\left(t_{2}, \ldots, t_{e(d)}\right)\right|=(q-1)^{n-1} q^{\operatorname{dim} \mathfrak{n}-(n-2+e(d))} .
$$

Since, $Z=\bigcup_{\left(t_{2}, \ldots, t_{e(d)}\right) \in k^{\times}} B \cdot \overline{\mathcal{F}}\left(t_{2}, \ldots, t_{e(d)}\right)$ is dense in $\mathfrak{n}$ and the $U$-orbit of each element of $Z$ has dimension $\operatorname{dim} \mathfrak{n}-(n-2+e(d))$, we can deduce as for the case $e(d)=1$ that $(q-1)^{n-1} q^{\operatorname{dim} \mathfrak{n}-(n-2+e(d))}$ is an upper bound for the size of a $B(q)$-orbit in $\mathfrak{n}(q)$.

\section{References}

[Bo] A. Borel, Linear algebraic groups, Graduate Texts in Mathematics 126, Springer-Verlag 1991. 
[BH1] T. Brüstle and L. Hille, Finite, Tame, and Wild Actions of Parabolic Subgroups in $\mathrm{GL}(V)$ on Certain Unipotent Subgroups. J. Algebra 226, no. 1 (2000), 347-360.

[BH2] T. Brüstle and L. Hille, Actions of Parabolic Subgroups in $\mathrm{GL}_{n}$ on Unipotent Normal Subgroups and Quasi-hereditary Algebras. Coll. Math. 83 (2000), 281-294.

[BHRR] T. Brüstle, L. Hille, C. M. Ringel and G. Röhrle, The $\Delta$-filtered Modules without Self-extensions for the Auslander Algebra of $k[T] /\left\langle T^{n}\right\rangle$. Algebr. Represent. Theory 2, no. 3 (1999), 295-312.

[BHRö] T. Brüstle, L. Hille and G. Röhrle, Finiteness results for parabolic group actions in classical groups, Arch. Math. 76(2) (2001), 81-87.

[BHRZ] T. Brüstle, L. Hille, G. Röhrle and G. Zwara, The Bruhat-Chevalley Order of Parabolic Group Actions in General Linear Groups and Degeneration for $\Delta$-filtered Modules. Adv. Math. 148, no. 2 (1999), 203242.

[BüHe] H. Bürgstein and W. H. Hesselink, Algorithmic orbit classification for some Borel group actions, Comp. Math. 61 (1987), 3-41.

[DR2] V. Dlab and C. M. Ringel, The Module Theoretical Approach to Quasihereditary Algebras. Representations of algebras and related topics (Kyoto, 1990), London Math. Soc. Lecture Note Ser. 168, Cambridge Univ. Press, Cambridge, 1992, 200-224.

[Gb] P. Gabriel, Finite representation type is open. Proceedings of the International Conference on Representations of Algebras (Carleton Univ., Ottawa, Ont., 1974), Paper No. 10, 23 pp. Carleton Math. Lecture Notes, No. 9, Carleton Univ., Ottawa, Ont., 1974.

[GbR] P. Gabriel and A. V. Roiter, Representations of Finite-dimensional Algebras. Springer-Verlag, Berlin, 1997.

[GS] C. Geiss and J. Schröer, Varieties of modules over tubular algebras. Coll. Math. 95 (2003), 163-183.

[Gd1] S. M. Goodwin, Algorithmic testing for dense orbits of Borel subgroups, J. Pure Appl. Algebra, 197 (2005), no. 1-3, 171-181.

[Gd2] S. M. Goodwin, On the conjugacy classes in maximal unipotent subgroups of simple algebraic groups, Transform. Groups, To appear (2004).

[GR1] S. M. Goodwin and G. Röhrle, Prehomogeneous spaces for parabolic group actions in classical groups, J. Algebra 276 (2004) no. 1, 383-398.

[GR2] S. M. Goodwin and G. Röhrle, Finite orbit modules for parabolic subgroups of exceptional groups, Indag. Math. 15 (2004), no. 2, 189-207. 
[He] W. Hesselink, Singularities in the nilpotent scheme of a classical group, Trans. Amer. Math. Soc. 222 (1976), 1-32.

[Hg] G. Higman, Enumerating p-groups. I: Inequalities, Proc. London Math. Soc. 3 (1960), no. 10, 24-30.

[Hl] L. Hille, Aktionen algebraischer Gruppen, geometrische Quotienten und Köcher. Habilitationsschrift, Hamburg 2003.

[HR2] L. Hille and G. Röhrle, A Classification of Parabolic Subgroups of Classical Groups with a Finite Number of Orbits on the Unipotent Radical. Transform. Groups 4, no. 1 (1999), 35-52.

[HR3] L. Hille and G. Röhrle, Variation on a Theme of Richardson. Linear Algebra Appl. 365 (2003), 239-246.

[JR] U. Jürgens and G. Röhrle, MOP - Algorithmic Modality Analysis for Parabolic Group actions, Experimental Math., 11 (2002) no. 1, 57-67.

[Rc] R. W. Richardson, Conjugacy Classes in Parabolic Subgroups of Semisimple Algebraic Groups. Bull. London Math. Soc. 6 (1974), 2124 .

[Rn] C. M. Ringel, Tame Algebras and Integral Quadratic Forms. Springer Lecture Notes in Math. 1099, Springer-Verlag, Berlin New York, 1984.

[Rb] G. R. Robinson, Counting conjugacy classes of unitriangular groups associated to finite-dimensional algebras, J. Group Theory 1 (1998), no. $3,271-274$.

[Th] J. Thompson, $k\left(\mathrm{U}_{n}\left(F_{q}\right)\right)$, Preprint, http://www.math.ufl.edu/fac/thompson.html.

[VA] A. Véra-Lopez and J.M. Arregi, Conjugacy classes in unitriangular matrices, Linear Algebra Appl. 370 (2003), 85-124.

Simon M. Goodwin

New College

Oxford

OX1 3LB

Email: simon.goodwin@new.ox.ac.uk

http://home.imf.au.dk/goodwin/

Lutz Hille

Mathematisches Seminar

Universität Hamburg

D-20 146 Hamburg

Email: hille@math.uni-hamburg.de

http://www.math.uni-hamburg.de/home/hille/ 